\title{
POLARIZATION AND POLARIMETRY: A REVIEW
}

\author{
SASCHA TRIPpE \\ Department of Physics and Astronomy, Seoul National University, Seoul 151-742, Korea \\ E-mail: trippe@astro.snu.ac.kr \\ (Received August 30, 2013; Accepted December 28, 2013)
}

\begin{abstract}
Polarization is a basic property of light and is fundamentally linked to the internal geometry of a source of radiation. Polarimetry complements photometric, spectroscopic, and imaging analyses of sources of radiation and has made possible multiple astrophysical discoveries. In this article I review (i) the physical basics of polarization: electromagnetic waves, photons, and parameterizations; (ii) astrophysical sources of polarization: scattering, synchrotron radiation, active media, and the Zeeman, GoldreichKylafis, and Hanle effects, as well as interactions between polarization and matter (like birefringence, Faraday rotation, or the Chandrasekhar-Fermi effect); (iii) observational methodology: on-sky geometry, influence of atmosphere and instrumental polarization, polarization statistics, and observational techniques for radio, optical, and $\mathrm{X} / \gamma$ wavelengths; and (iv) science cases for astronomical polarimetry: solar and stellar physics, planetary system bodies, interstellar matter, astrobiology, astronomical masers, pulsars, galactic magnetic fields, gamma-ray bursts, active galactic nuclei, and cosmic microwave background radiation.
\end{abstract}

Key words : Polarization — Methods: polarimetric — Radiation mechanisms: general

\section{Contents}

1. INTRODUCTION . . . . . . . . . . 16

2. PHYSICAL BASICS . . . . . . . . . . . 16

2.1. Electromagnetic Waves . . . . . . . 16

2.1.1. Electric Field Vectors . . . . . 16

2.1.2. Elliptical Polarization . . . . . 16

2.1.3. Linear Polarization . . . . . . . 16

2.1.4. Circular Polarization . . . . . . 17

2.1.5. Macroscopic Polarization . . . . 17

2.2. Photons . . . . . . . . . . . . . 17

2.3. Parameterizations . . . . . . . . . . 18

2.3.1. Jones Calculus . . . . . . . . 18

2.3.2. Stokes Parameters . . . . . . 19

2.3.3. Müller Formalism . . . . . . . . . 20

3. POLARIGENESIS . . . . . . . . . . . . 20

3.1. Scattering Polarization . . . . . . . . 21

3.1.1. Microscopic Scattering . . . . . 21

3.1.2. Scattering by Dust . . . . . . . 21

3.2. Dichroic Media . . . . . . . . . . . 21

3.3. Optically Active Media . . . . . . . . . . . 22

3.4. Synchrotron Radiation . . . . . . . . . 22

3.5. Zeeman Effect . . . . . . . . . . . . 23

3.6. Goldreich-Kylafis Effect . . . . . . . . . . 24

3.7. Hanle Effect . . . . . . . . . . . . . . . . . 24

3.8. Interactions of Polarization and Matter . . 25

3.8.1. Partial Reflection . . . . . . . . 25

3.8.2. Birefringence . . . . . . . . . 25

3.8.3. Faraday Rotation . . . . . . 26
3.8.4. Faraday Depolarization . . . . 26

3.8.5. Polarization Conversion . . . . . 27

3.8.6. Chandrasekhar-Fermi Effect . . . 27

4. OBSERVATIONS . . . . . . . . . 27

4.1. Sky Projection . . . . . . . . . . . . . 27

4.2. Terrestrial Atmosphere . . . . . . . . . . . 28

4.3. Instrumental Polarization . . . . . . . . 28

4.4. Polarization Statistics . . . . . . . . . . . 28

4.5. Radio Observations . . . . . . . . . . . . . 29

4.6. Optical Observations . . . . . . . . . . . . 29

4.7. X and $\gamma$ Ray Observations . . . . . . . . 30

5. SCIENCE CASES .............. . . 31

5.1. Solar and Stellar Physics . . . . . . . . . 31

5.2. Planetary System Bodies . . . . . . . . . . 32

5.2.1. Solid Surfaces . . . . . . . . . . 32

5.2.2. Atmospheres . . . . . . . . . . . . . 32

5.3. Interstellar Matter . . . . . . . . . . . . . . . . . . 32

5.4. Astrobiology . . . . . . . . . . . . . 33

5.5. Astronomical Masers . . . . . . . . . . . . 33

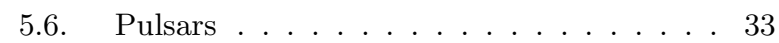

5.7. Active Galactic Nuclei . . . . . . . . . . 34

5.8. Galactic Magnetic Fields . . . . . . . . . . 34

5.9. Gamma Ray Bursts . . . . . . . . . . 35

5.10. Cosmic Background Radiation . . . . . 35

6. CONCLUSIONS .................. 36

Acknowledgments . . . . . . . . . . . . 36

References . . . . . . . . . . . . 36 


\section{INTRODUCTION}

"To many astrophysicists, stellar polarimetry is a Cinderella subject considered as being so insignificant and, at the same time, being so esoteric as to be ignored and left alone. [...] There can be no doubt, however, that the study of polarization within astronomy has a strong role to play either in its own right, or in combination with other observational tools, as a diagnostic for understanding the behaviour of celestial sources."

- Clarke (2010), p. XIII

Historically, the study of polarized light began with the discovery of birefringence in crystals by Erasmus Bartholinus and its subsequent interpretation by Christian Huygens around the year 1670 (Brosseau 1998). Astronomical observations of polarized light commenced in the middle of the 19th century; some of the earliest publications treat the linear polarization of sun light reflected by the moon (Secchi 1860) and the linear polarization of the light from the solar corona (Edlund 1860). Subsequently, the field of polarimetry evolved closely with the technical progress of observational techniques in general: from optical polarimetry to radio polarimetry in the 1940s (Wilson, Rohlfs, \& Hüttemeister 2010) and eventually to space-based Xray polarimetry in the 1970s (Weisskopf et al. 1978).

Polarization is a fundamental property of electromagnetic radiation. It is a rich source of information on the physical properties - magnetic fields, internal conditions, particle densities, et cetera - of astronomical objects. Polarimetric observations complement analysis methods based on photometry as well as on spectral (spectroscopy) or angular (mapping, imaging) resolution. Accordingly, polarimetry has contributed substantially to the progress of astronomy. Milestones have been, for example:

- the mapping of solar and stellar magnetic fields (e.g., Schrijver \& Zwaan 2000);

- the characterization of the surface composition of solar system bodies (e.g., Bowell \& Zellner 1974);

- the discovery of synchrotron radiation from astronomical objects (Oort \& Walraven 1956);

- the discovery and characterization of large-scale (many kpc) galactic magnetic fields (e.g., Kulsrud \& Zweibel 2008);

- the analysis of the polarization modes of the cosmic microwave background (e.g., Kovac et al. 2002).

This review provides a broad overview on the theory and phenomenology of polarization in astronomy. It covers (i) the physical basics of polarized radiation, (ii) sources of astrophysical polarization, (iii) concepts and methods of astronomical polarimetry, and (iv) astrophysical science cases.

\section{PHYSICAL BASICS}

\subsection{Electromagnetic Waves}

\subsubsection{Electric Field Vectors}

The concept of electromagnetic waves derives from nonzero solutions of Maxwell's equations in vacuum, meaning here specifically the absence of electric charges (e.g., Landau \& Lifschitz 1997; Jackson 1999). Following common conventions, we regard the electric field* $\mathbf{E}$ of an electromagnetic wave traveling in $z$ direction in Euclidean coordinates $(x, y, z)$ with speed of light $c$. Accordingly, we have - in trigonometric notation -

$$
\mathbf{E}(t, z)=\mathbf{E}(0,0) \cos (\omega t-k z-\phi)
$$

where $t$ is the time, $\omega$ denotes the angular frequency, $k=\omega / c$ is the absolute value of the wave vector, and $\phi$ denotes an arbitrary phase. As the electric field vector is perpendicular to $z$, we can decompose $\mathbf{E}(t, z)$ into its $x$ and $y$ components. For simplicity, we regard the location $z=0$ only, i.e., we regard the location of $\mathbf{E}(t)$ in the $x y$ plane. The $x$ and $y$ components are then

$$
\begin{aligned}
& E_{x}(t)=E_{x}(0) \cos \left(\omega t-\phi_{1}\right) \\
& E_{y}(t)=E_{y}(0) \cos \left(\omega t-\phi_{2}\right) .
\end{aligned}
$$

Here, $\phi_{1,2}$ denote two - a priori arbitrary - phases. In addition, we denote the angle between $\mathbf{E}(t)$ and the positive $x$ axis - the polarization angle, counted in counterclockwise direction - with $\chi$. The polarization of the wave is given by the relative values of $E_{x}(0), E_{y}(0), \phi_{1}$, and $\phi_{2}$ (Rybicki \& Lightman 1979; Huard 1997; Landau \& Lifschitz 1997; Jackson 1999; Born \& Wolf 1999; Goldstein 2003).

\subsubsection{Elliptical Polarization}

In general, the tip of the electric field vector follows an elliptical trajectory in the $x y$ plane; accordingly, the light is denoted as elliptically polarized. The orientation of the ellipse in the $x y$ plane is constant in time; the polarization angle $\chi$ corresponds to the angle between the positive $x$ axis and the semi-major axis of the ellipse (counted in counterclockwise direction).

Elliptical polarization is the most general state of polarization of an electromagnetic wave. Linear polarization occurs if the polarization ellipse degenerates into a line. Circular polarization corresponds to the opposite - special case of the ellipse degenerating into a circle.

\subsubsection{Linear Polarization}

For the case $\phi_{1}=\phi_{2}$, using here specifically $\phi_{1}=\phi_{2}=$ 0 without loss of generality, we have

* The magnetic field is perpendicular to the direction of travel and to the electric field. The amplitude of the magnetic field, $B$, is related to the amplitude of the electric field, $E$, like $B=E / c$. 


$$
\begin{aligned}
& E_{x}(t)=E_{x}(0) \cos (\omega t) \\
& E_{y}(t)=E_{y}(0) \cos (\omega t) .
\end{aligned}
$$

The orientation of $\mathbf{E}$ then depends only on the magnitudes of $E_{x}(0)$ and $E_{y}(0)$ and is independent of time; the angle $\chi$ is constant. The radiation is linearly polarized with polarization angle $\chi \in[0, \pi]$. The orientation of the plane wherein the wave is located - the plane of polarization - as given by $\chi$ has an orientation but no direction; accordingly, the location of linear polarization in the $x y$ plane is not a vector. In physics and astronomy, the $x$ and $y$ components of linearly polarized light are commonly identified with horizontal $(\mathrm{H})$ and vertical $(\mathrm{V})$ polarizations, respectively.

\subsubsection{Circular Polarization}

In case of a relative phase shift $\phi_{2}=\phi_{1} \pm \pi / 2$, using here specifically $\phi_{1}=0$ without loss of generality, and $E_{x}(0)=E_{y}(0)$, we have

$$
\begin{aligned}
& E_{x}(t)=E_{x}(0) \cos (\omega t) \\
& E_{y}(t)= \pm E_{y}(0) \sin (\omega t) .
\end{aligned}
$$

The tip of the electric field vector moves circularly in the $x y$ plane with angular frequency $\omega$ : the radiation is circularly polarized. The sign of $E_{y}(t)$ - which derives from the relative phase - determines the sense of the motion of $\mathbf{E}$. A positive sign, corresponding to counterclockwise motion, is commonly referred to as right-hand circular (RHC) polarization. Accordingly, a negative sign, corresponding to clockwise motion, is denoted as left-hand circular (LHC) polarization.

\subsubsection{Macroscopic Polarization}

An individual electromagnetic wave is necessarily polarized as described above (microscopic polarization). Astrophysical observations do not deal with individual waves but with radiation that is a (almost always incoherent) superposition of a very large number of elementary electromagnetic waves. Accordingly, astronomical observations are sensitive to the macroscopic polarization of light. For most physical systems, all orientations of the electric field vectors from the elementary emitters are equally probable - there is, a priori, no reason to expect a macroscopic polarization of light. Confusingly, unpolarized light is therefore sometimes referred to as "natural" light.

A (macroscopic) polarization signal (Fowles 1975; Rybicki \& Lightman 1979; Mandel \& Wolf 1995; Born \& Wolf 1999) can occur whenever the internal geometry of the source of radiation or the properties of the interstellar medium prefer a certain orientation of electric field vectors. In those cases - discussed in detail in Section 3 - the light becomes partially polarized with a degree of polarization

$$
m_{P}=\frac{I_{P}}{I} \in[0,1]
$$

where $I$ denotes the total intensity of the light and $I_{P}$ denotes the intensity of polarized light..$^{\dagger}$ Intensity $I$ and amplitude of electric field $E$ are related like $I \propto E^{2}$. The total polarization state of radiation can be described as a superposition of linear and circular polarization (cf., Section 2.1.2-2.1.4); accordingly, we can define separate degrees of linear and circular polarization. The degree of linear polarization is given by

$$
m_{L}=\frac{I_{L}}{I} \in[0,1]
$$

where $I_{L}$ denotes the intensity of linearly polarized light. The degree of circular polarization is given by

$$
m_{C}=\frac{I_{C}}{I} \in[-1,1]
$$

where $I_{C}$ denotes the intensity of circularly polarized light. The sign of $I_{C}$ and thus $m_{C}$ depends on the orientation of the polarization. By convention, the positive (negative) sign is assigned to light with $I_{\mathrm{RHC}}-I_{\mathrm{LHC}}>0(<0)$; here, $I_{\mathrm{RHC}}$ and $I_{\mathrm{LHC}}$ denote the intensities of right-hand circularly and left-hand circularly polarized light, respectively (Hamaker \& Bregman 1996).

\subsection{Photons}

The wave-particle dualism of light (Einstein 1905) implies that polarization is a property of individual photons; each photon can be assigned an individual state of polarization (Dirac 1958).

Using the standard bra-ket notation for quantum states, ${ }^{\ddagger}$ we may write two arbitrary photon states like $|X\rangle$ and $|Y\rangle$. These two states are orthogonal if $\langle X \mid Y\rangle=0$. We may further assume that all states are normalized, meaning $\langle X \mid X\rangle=1$ for arbitrary $X$. The bracket product $\langle X \mid Y\rangle$ is the probability amplitude of the event "The system in state $X$ is also in state $Y$ ", and $|\langle X \mid Y\rangle|^{2} \in[0,1]$ is the corresponding probability; $|. .$.$| denotes the absolute value of the enclosed function.$

For the specific case of photon polarization (Bachor \& Ralph 2004), we have to consider photon states corresponding to horizontal linear polarization $|H\rangle$, vertical linear polarization $|V\rangle$, right-hand circular polarization

\footnotetext{
${ }^{\dagger}$ In the astronomical literature, degrees of polarization are commonly quoted in units of per cent (\%).

$\ddagger$ One may picture bras $\langle X|$ as complex row vectors and kets $|X\rangle$ as complex column vectors of equal - potentially infinite - dimension. A bra transforms into a ket by transposition plus complex conjugation (Hermitian conjugate). Accordingly, the product $|X\rangle\langle Y|$ corresponds to a complex matrix, the product $\langle X \mid Y\rangle$ corresponds to a complex scalar (e.g., Dirac 1958).
} 
$|R\rangle$, and left-hand circular polarization $|L\rangle .^{\S}$ Evidently, $|H\rangle$ and $|V\rangle$ on the one hand and $|R\rangle$ and $|L\rangle$ on the other hand are mutually exclusive, meaning

$$
\begin{aligned}
& \langle H \mid V\rangle=\langle V \mid H\rangle=0 \\
& \langle R \mid L\rangle=\langle L \mid R\rangle=0 \text {. }
\end{aligned}
$$

Due to normalization, we further have

$$
\begin{array}{r}
\langle H \mid H\rangle=\langle V \mid V\rangle=1 \\
\langle R \mid R\rangle=\langle L \mid L\rangle=1
\end{array}
$$

In addition, we have to note the relations between linear and circular polarization states. A standard tool employed in quantum optics is a polarizing beamsplitter that is sensitive to linear polarization: in a (thought) laboratory experiment, incident photons in state $|H\rangle$ are sent into one direction, photons in state $|V\rangle$ into another. The beam-splitter is insensitive to circular polarization; an incident photon in state $|R\rangle$ or $|L\rangle$ is sent into either direction with equal probability of $50 \%$. Accordingly, one finds

$$
\begin{aligned}
& |\langle R \mid V\rangle|^{2}=|\langle R \mid H\rangle|^{2}=0.5 \\
& |\langle L \mid V\rangle|^{2}=|\langle L \mid H\rangle|^{2}=0.5
\end{aligned}
$$

Combining the information from Eqs. 8, 9, and 10, it turns out (Bachor \& Ralph 2004) that circular polarization states can be expressed as superpositions of linear polarization states like

$$
\begin{aligned}
|R\rangle & =\frac{1}{\sqrt{2}}(|H\rangle+i|V\rangle) \\
|L\rangle & =\frac{1}{\sqrt{2}}(|H\rangle-i|V\rangle) .
\end{aligned}
$$

with $i$ being the imaginary unit. An arbitrary polarization state $|P\rangle$ can be expressed as

$$
|P\rangle=a|H\rangle+e^{i \phi} b|V\rangle
$$

with $\phi, a$, and $b$ being real numbers, and $a^{2}+b^{2}=1$. The representation given by Eq. 12 is not unique; any pair of orthogonal states can be used as base vectors.

In analogy to the case of electromagnetic waves, the macroscopic polarization of light is given by the superposition of a large number of photons with individual microscopic polarization states. The discussion provided in Section 2.1.5 is equally valid for waves and particles.

\footnotetext{
$\S$ Only up to this sub-section $V$ denotes vertical linear polarization. In the remainder of this paper, $V$ denotes the corresponding Stokes parameter (Section 2.3.2).
}

\subsection{Parameterizations}

\subsubsection{Jones Calculus}

In Eq. 2, I introduced the components of the electric field in trigonometric notation for convenience. Likewise, the electric field can be given in complex exponential notation as

$$
\begin{aligned}
& E_{x}(t)=E_{x}(0) e^{i\left(\omega t-\phi_{1}\right)} \\
& E_{y}(t)=E_{y}(0) e^{i\left(\omega t-\phi_{2}\right)} .
\end{aligned}
$$

One may now define (Jones 1941; Fowles 1975; Huard 1997; Goldstein 2003) the Jones vector

$$
\mathbf{e} \equiv\left[\begin{array}{c}
E_{x}(0) e^{i \phi_{1}} \\
E_{y}(0) e^{i \phi_{2}}
\end{array}\right]
$$

that expresses amplitudes and phases of the electric field in vector form. A convenient - usually not normalized - form of the Jones vector is achieved by expressing the components in units of the amplitude of one of them. Linearly polarized waves may be expressed like

$$
\mathbf{e}_{x}=\left[\begin{array}{l}
1 \\
0
\end{array}\right] ; \quad \mathbf{e}_{y}=\left[\begin{array}{l}
0 \\
1
\end{array}\right]
$$

where $\mathbf{e}_{x}$ and $\mathbf{e}_{y}$ denote waves polarized in $x$ and $y$ direction, respectively. Likewise, by exploiting the identity $\pm i=e^{ \pm i \pi / 2}$, circularly polarized waves can be expressed like

$$
\mathbf{e}_{L}=\left[\begin{array}{c}
1 \\
i
\end{array}\right] ; \quad \mathbf{e}_{R}=\left[\begin{array}{c}
1 \\
-i
\end{array}\right]
$$

where $\mathbf{e}_{L}$ and $\mathbf{e}_{R}$ denote left-hand and right-hand circular polarization, respectively. The result of a superposition of electric fields is given by the sum of the appropriate Jones vectors. A noteworthy example is

$$
\left[\begin{array}{l}
1 \\
i
\end{array}\right]+\left[\begin{array}{c}
1 \\
-i
\end{array}\right]=\left[\begin{array}{l}
2 \\
0
\end{array}\right]=2\left[\begin{array}{l}
1 \\
0
\end{array}\right]
$$

which demonstrates that a linearly polarized wave can be expressed as the sum of a left-hand and a right-hand circularly polarized wave with equal amplitudes - as demanded by equivalence with Eqs. 11, 12. In general, any polarization state can be expressed as combination of two Jones vectors $\mathbf{e}_{1}, \mathbf{e}_{2}$ that represent orthogonal polarizations, meaning

$$
\mathbf{e}_{1} \mathbf{e}_{2}^{*}=0
$$

where the operator * denotes complex conjugation.

A (linear) modification of the polarization state of a wave is expressed by a $2 \times 2$ Jones matrix $\mathbf{J}$ that relates input wave $\mathbf{e}$ and output wave $\mathbf{e}^{\prime}$ like

$$
\mathbf{e}^{\prime}=\mathbf{J e}
$$



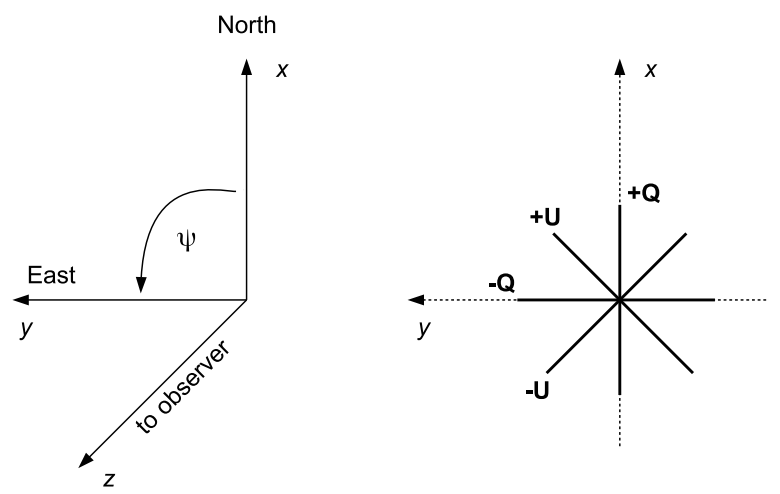

Fig. 1. - Fundamental coordinates and geometries of the Stokes parameters $Q$ and $U ; \psi$ denotes the parallactic angle.

In optics, Jones matrices are commonly employed to characterize polarizing optical elements or trains thereof. Successive modifications $1,2, \ldots, n$ of the polarization state can be written in terms of a single Jones matrix. This matrix is given by the product of the matrices corresponding to the individual optical elements like

$$
\mathbf{J}=\mathbf{J}_{n} \mathbf{J}_{n-1} \ldots \mathbf{J}_{2} \mathbf{J}_{1}
$$

Simple examples for Jones matrices of polarizing optical elements are

$$
\mathbf{J}_{x}=\left[\begin{array}{ll}
1 & 0 \\
0 & 0
\end{array}\right] ; \quad \mathbf{J}_{R}=\frac{1}{2}\left[\begin{array}{rr}
1 & i \\
-i & 1
\end{array}\right]
$$

where $\mathbf{J}_{x}$ describes a linear polarizer with the $x$ axis being the transmission axis, and $\mathbf{J}_{R}$ corresponds to a right-hand circular polarizer.

\subsubsection{Stokes Parameters}

We understand from Section 2.1.2-2.1.4 that the polarization state of an electromagnetic wave can be characterized by means of three independent parameters: the amplitudes $E_{x}(0)$ and $E_{y}(0)$ and the phase difference $\delta=\phi_{2}-\phi_{1}$. In general, astronomical observations deal with light intensities rather than with field amplitudes; accordingly, it is convenient to quantify polarization via characteristic intensities. For reasons that are going to be evident soon, those characteristic intensities $\pi$ are the Stokes parameters"

IIn the convention we adopt here, intensity $I$ and field amplitude $E$ are related like $I=E^{2}$, whereas in SI units $I=\varepsilon_{0} c E^{2}$ with vacuum permittivity $\varepsilon_{0}$. Accordingly, our convention implies a rescaling of electric fields like $E \longrightarrow E^{\prime}=\sqrt{\varepsilon_{0} c} E$

" Occasionally, the parameters $I, Q, U, V$ are also denoted as $S_{0}$, $S_{1}, S_{2}, S_{3}$, respectively.

$$
\begin{aligned}
I & =\left\langle E_{x}^{2}\right\rangle+\left\langle E_{y}^{2}\right\rangle \\
Q & =\left\langle E_{x}^{2}\right\rangle-\left\langle E_{y}^{2}\right\rangle \\
U & =2\left\langle E_{x} E_{y} \cos \delta\right\rangle \\
V & =2\left\langle E_{x} E_{y} \sin \delta\right\rangle
\end{aligned}
$$

(Stokes 1852) where $E_{x, y} \equiv E_{x, y}(t)$ for simplicity, and $\langle\ldots\rangle$ denotes the time average of the enclosed parameters taken over times much larger than $2 \pi / \omega$ (Huard 1997; Born \& Wolf 1999; Goldstein 2003; Thompson, Moran, \& Swenson 2004; Wilson, Rohlfs, \& Hüttemeister 2010). Notably, the parameters $Q, U$, and $V$ can take negative values.

By construction, $I$ is the intensity of the wave. The parameter $Q$ quantifies a difference in the intensities in $x$ and $y$, thus providing information on linear polarization. The parameter $U$ quantifies the difference between the two field components diagonal - at angles of $45^{\circ}$ and $135^{\circ}$ counted from the positive $x$ axis - to the $x$ and $y$ coordinates, thus likewise probing linear polarization. Finally, the parameter $V$ corresponds to the circularly polarized intensity. An illustration of the fundamental geometries is provided in Fig. 1.

For individual waves - microscopic polarization the Stokes parameters are related via

$$
I^{2}=Q^{2}+U^{2}+V^{2},
$$

thus reducing the number of free parameters to three - as expected. As $I$ is a constant, each polarization state of a wave corresponds to a point on a sphere, the Poincaré sphere (Poincaré 1892). In case of macroscopic polarization, radiation with intensity $I$ is formed from superposition of many elementary emitters; polarization is averaged out at least partially. Accordingly, Eq. 23 breaks down to

$$
I_{P}^{2}=Q^{2}+U^{2}+V^{2}
$$

with $I_{P} \leq I$ being the polarized intensity; the number of free parameters increases to four, the fourth parameter being $I$. Using the definitions provided by Eqs. 5, 6 , and 7 as well as the definition of the polarization angle $\chi$, the Stokes parameters relate to the parameters of linear polarization like

$$
\begin{aligned}
m_{L}=\frac{\sqrt{Q^{2}+U^{2}}}{I} & \in[0,1] \\
\chi=\frac{1}{2} \operatorname{atan}_{2}\left(\frac{U}{Q}\right) & \in[0, \pi]
\end{aligned}
$$

where $\operatorname{atan}_{2}$ denotes the quadrant-preserving arc tangent; $m_{L}$ and $\chi$ correspond to the length and the orientation of a vector centered at the origin of a plane spanned by $Q$ and $U$. The degree of circular polarization relates to Stokes $V$ like 


$$
m_{C}=\frac{V}{I} \quad \in[-1,1] .
$$

When using complex exponential notation for the electric field (Eq. 13), we find an alternative - though equivalent - definition of the Stokes parameters:

$$
\begin{aligned}
I & =\left\langle E_{x} E_{x}^{*}\right\rangle+\left\langle E_{y} E_{y}^{*}\right\rangle \\
Q & =\left\langle E_{x} E_{x}^{*}\right\rangle-\left\langle E_{y} E_{y}^{*}\right\rangle \\
U & =\left\langle E_{x} E_{y}^{*}\right\rangle+\left\langle E_{y} E_{x}^{*}\right\rangle \\
V & =-i\left[\left\langle E_{x} E_{y}^{*}\right\rangle-\left\langle E_{y} E_{x}^{*}\right\rangle\right] .
\end{aligned}
$$

As usual, the operator * denotes complex conjugation. It is straightforward to see that this definition is equivalent to Eq. 22 (e.g., Hamaker \& Bregman 1996).

In astronomy, the Euclidean coordinates we use here are conventionally defined such that the $x$ axis points to the north, the $y$ axis points to the east, and the $z$ axis points toward the observer. Accordingly, the polarization angle $\chi$ is counted from north to east. The orientations of circular polarizations as defined in Section 2.1.4 - RHC and LHC - are preserved.

As I indicated before in Section 2.2 and Section 2.3.1, the polarization state of photons as well as electromagnetic waves can be described using left- and right-hand circular polarization components with amplitudes $E_{L, R}$ and phases $\phi_{L, R}$ as base. Using $\delta^{\prime}=$ $\phi_{R}-\phi_{L}$, we find (e.g., Cenacchi et al. 2009) for the Stokes parameters in trigonometric notation

$$
\begin{aligned}
I & =\left\langle E_{R}^{2}\right\rangle+\left\langle E_{L}^{2}\right\rangle \\
Q & =2\left\langle E_{R} E_{L} \cos \delta^{\prime}\right\rangle \\
U & =2\left\langle E_{R} E_{L} \sin \delta^{\prime}\right\rangle \\
V & =\left\langle E_{R}^{2}\right\rangle-\left\langle E_{L}^{2}\right\rangle
\end{aligned}
$$

and in complex exponential notation

$$
\begin{aligned}
I & =\left\langle E_{R} E_{R}^{*}\right\rangle+\left\langle E_{L} E_{L}^{*}\right\rangle \\
Q & =\left\langle E_{R} E_{L}^{*}\right\rangle+\left\langle E_{L} E_{R}^{*}\right\rangle \\
U & =-i\left[\left\langle E_{R} E_{L}^{*}\right\rangle-\left\langle E_{L} E_{R}^{*}\right\rangle\right] \\
V & =\left\langle E_{R} E_{R}^{*}\right\rangle-\left\langle E_{L} E_{L}^{*}\right\rangle .
\end{aligned}
$$

It is important to note that - in general - the treatment of combinations of polarized signals requires the use of the Stokes parameters: only intensities can be added or subtracted in a straightforward manner - degrees of polarization or polarization angles cannot (cf., e.g., Heiles 2002).

\subsubsection{Müller Formalism}

The Müller formalism (Müller 1948; Hamaker, Bregman, \& Sault 1996; Huard 1997; Goldstein 2003) ex- tends and combines Jones calculus and Stokes formalism. The Stokes parameters can be expressed as a fourdimensional vector, the Stokes vector

$$
\mathbf{S}=\left[\begin{array}{c}
I \\
Q \\
U \\
V
\end{array}\right] \equiv \mathbf{T} \mathbf{C}
$$

with

$$
\mathbf{C}=\left[\begin{array}{c}
\left\langle E_{x} E_{x}^{*}\right\rangle \\
\left\langle E_{x} E_{y}^{*}\right\rangle \\
\left\langle E_{y} E_{x}^{*}\right\rangle \\
\left\langle E_{y} E_{y}^{*}\right\rangle
\end{array}\right] ; \mathbf{T}=\left[\begin{array}{rrrr}
1 & 0 & 0 & 1 \\
1 & 0 & 0 & -1 \\
0 & 1 & 1 & 0 \\
0 & -i & i & 0
\end{array}\right]
$$

The vector $\mathbf{C}$ is commonly referred to as coherency vector; evidently, this notation is equivalent to Eq. 27.

Modifications of the polarization state can be expressed as modifications of the Stokes vector like

$$
\mathbf{S}^{\prime}=\mathbf{M S}
$$

where $\mathbf{M}$ is a $4 \times 4$ Müller matrix. Simple examples are

$$
\mathbf{M}_{\mathrm{ref}}=\left[\begin{array}{rrrr}
1 & 0 & 0 & 0 \\
0 & 1 & 0 & 0 \\
0 & 0 & -1 & 0 \\
0 & 0 & 0 & -1
\end{array}\right]
$$

and

$$
\mathbf{M}_{\mathrm{rot}}=\left[\begin{array}{rrrr}
1 & 0 & 0 & 0 \\
0 & \cos 2 \beta & \sin 2 \beta & 0 \\
0 & -\sin 2 \beta & \cos 2 \beta & 0 \\
0 & 0 & 0 & 1
\end{array}\right]
$$

that correspond to a reflection at a mirror and rotation by an angle $\beta$, respectively.

Else than the Jones calculus, the Müller formalism can describe unpolarized light as well as depolarization, i.e., a reduction of the degree of (total) polarization $m_{P}$.

\section{POLARIGENESIS}

As I discussed briefly in Section 2.1.5, the occurrence of a macroscopic polarization of light - the polarigenesis - is intimately linked to the internal symmetry of the physical system under consideration. Macroscopic polarization requires that the internal structure of the source of light is anisotropic. We can identify a variety of astrophysical sources of polarized light that I discuss in the following. 


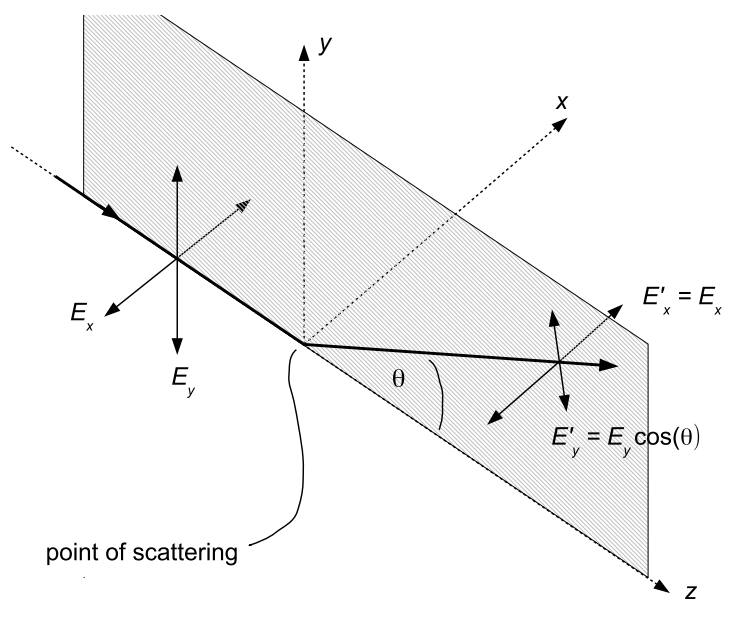

Fig. 2. - The geometry of scattering polarization. An unpolarized light ray with linear polarization components $E_{x, y}$ propagates in positive $z$ direction. The ray is scattered by an angle $\theta$ and continues to propagate in the $y z$ plane (shaded). From the point of view of an observer of the scattered light, the polarization components perpendicular to the direction of propagation are $E_{x}^{\prime}=E_{x}$ and $E_{y}^{\prime}=$ $E_{y} \cos \theta$, respectively: the light is linearly polarized.

\subsection{Scattering Polarization}

\subsubsection{Microscopic Scattering}

Microscopic scattering processes - meaning the scattering of a photon at a free electric charge like an electron, an atom, or a molecule - lead to a characteristic linear polarization of the scattered light. Notably, the following geometry argument holds for a variety of scattering processes like Thomson scattering, Compton scattering, Rayleigh scattering, fluorescence, or Raman scattering regardless of the different underlying physical mechanisms. From now on, I assume incident unpolarized light propagating in $z$ direction with electric field components $E_{x, y}$ in $x$ and $y$ directions. Upon interaction with a charge, atom, or molecule, the light is scattered and continues to propagate in the $y z$ plane at an angle $\theta$ to the $z$ axis.

From the point of view of an observer of the scattered light, the light intensities in $x$ direction are the same before and after the scattering, i.e. $E_{x}^{\prime 2}=E_{x}^{2}$, with the prime denoting the scattered light. The $y$ component transforms like $E_{y}^{\prime 2}=E_{y}^{2} \cos ^{2} \theta$, meaning the intensity is reduced by a factor $\cos ^{2} \theta$. This implies that the scattered light is linearly polarized with a degree of polarization

$$
m_{L}=\frac{I_{x}^{\prime}-I_{y}^{\prime}}{I_{x}^{\prime}+I_{y}^{\prime}}=\frac{1-\cos ^{2} \theta}{1+\cos ^{2} \theta} \in[0,1]
$$

with $I_{x, y}^{\prime}$ denoting the observed intensities (after the scattering); I provide an illustration in Fig. 2.

\subsubsection{Scattering by Dust}

Dust grains are an important ingredient of interstellar matter (Dyson \& Williams 1997; Kwok 2007). Grain sizes are typically on the order of a micrometer, approximately corresponding to the wavelengths of optical to infrared light. A quantitative description of scattering of light by dust is provided by Mie's theory that assumes scattering by small spherical particles. From geometrical arguments equivalent to those presented in Section 3.1.1 one finds that initially unpolarized incident light becomes linearly polarized by dust scattering; the degree of polarization is given by an expression equivalent to Eq. 35 (Born \& Wolf 1999).

The derivation of Eq. 35 assumes that the incident light is propagating along a single well-defined direction. In clouds of interstellar matter, this is usually not the case: dust clouds tend to be optically thick, meaning that incident light experiences multiple scattering, absorption, and re-emission events, making the radiation field within the cloud isotropic. In this case, linear polarization can occur if (i) the dust grains have elongated (cylindrical, ellipsoidal) shapes, and (ii) the grains are oriented collectively along a preferred direction by magnetic fields. The absorption of radiation by the dust becomes a function of orientation relative to the magnetic field - resulting in linear polarization. Empirically, it has been found (Serkowski, Mathewson, \& Ford 1975; Draine 2003) that at optical to nearinfrared wavelengths the linear polarization is

$$
\frac{m_{L}}{m_{L}^{\max }} \approx \exp \left[-1.15 \ln ^{2}\left(\frac{\lambda_{\max }}{\lambda}\right)\right]
$$

scaled by the degree of linear polarization at a reference wavelength,

$$
m_{L}^{\max } \lesssim 0.03 A\left(\lambda_{\max }\right)
$$

where $\lambda$ is the wavelength, $\lambda_{\max } \approx 550 \mathrm{~nm}, A$ is the extinction in units of photometric magnitudes, and ln denotes the logarithm to base $e$; this relation is commonly referred to as Serkowski's law.

\subsection{Dichroic Media}

Dichroism is a further effect of the electric anisotropy of certain materials. Here the attenuation of light due to absorption by the material is anisotropic. Assuming initially unpolarized light, one component of the wave experiences stronger attenuation than the other one; the light becomes polarized.** Depending on if the difference in absorption affects the linear or circular wave components, the medium is referred to as linear dichroic or circular dichroic, respectively. Accordingly,

** The term "dichroism" is actually misleading. Historically, the first dichroic crystals studied showed a strong dependence of the effect on the wavelength of the light, leading to rays with different polarization having different colors. 
the light becomes either linearly or circularly polarized. The most efficient linear dichroic polarizers are polaroids, sheets of organic polymers with long-chain molecules which are aligned by stretching (Huard 1997; Born \& Wolf 1999).

In the presence of a magnetic field, a plasma becomes dichroic with respect to circular polarization. Assuming a propagation of light along the magnetic field lines, the ratio of the absorption coefficients for LHC and RHC polarized light, $\kappa_{L}$ and $\kappa_{R}$, respectively, is

$$
\frac{\kappa_{L}}{\kappa_{R}}=\left(\frac{\omega+\omega_{B}}{\omega-\omega_{B}}\right)^{2}
$$

where $\omega$ denotes the (angular) frequency of the light and $\omega_{B}$ denotes the (angular) gyration frequency of charged particles (usually electrons). This relation assumes $\omega_{B} \ll \omega$; the resulting circular polarization is $m_{C}=2 \omega_{B} / \omega$ (Angel 1974).

\subsection{Optically Active Media}

Materials composed of helically shaped molecules affect the polarization state of reflected or transmitted light very similar to birefringent and/or dichroic media (Sections 3.2 and 3.8.2). Macroscopic polarization arises if one of the two possible helix orientations is preferred; this is the case in a variety of biological materials. Initially unpolarized light reflected from helically layered surface structures on some insects can reach circular polarizations up to $m_{C} \approx 100 \%$ (Wolstencroft 1974).

\subsection{Synchrotron Radiation}

Synchrotron radiation is arguably the most important type of non-thermal continuum radiation from astronomical sources. It is emitted by electric charges usually electrons - gyrating around magnetic field lines at relativistic velocities. Assuming a magnetic field directed in $z$ direction, the magnetic Lorentz force enforces a circular motion in the $x y$ plane. In addition, the electron will usually have a non-zero velocity in $z$ direction, meaning that the overall trajectory of the electron has a helical shape. From the point of view of an external, not co-moving, observer the radiation is emitted from the electron in forward direction into a narrow cone with half opening angle $\theta \approx 1 / \gamma$, with $\gamma$ being the relativistic Lorentz factor ${ }^{\dagger \dagger}$ (Ginzburg \& Syrovatskii 1965; Rybicki \& Lightman 1979; Bradt 2008). Due to geometry, the observer sees the orbit of the electron as an ellipse in projection - the radiation emitted by a single oscillating charge is thus elliptically polarized.

Collimated electron beam. Macroscopically, the amounts of linear and circular polarization observed by

\footnotetext{
†† $\gamma=\left(1-v^{2} / c^{2}\right)^{-1 / 2}$, with $v$ being the electron speed and $c$ being the speed of light.
}

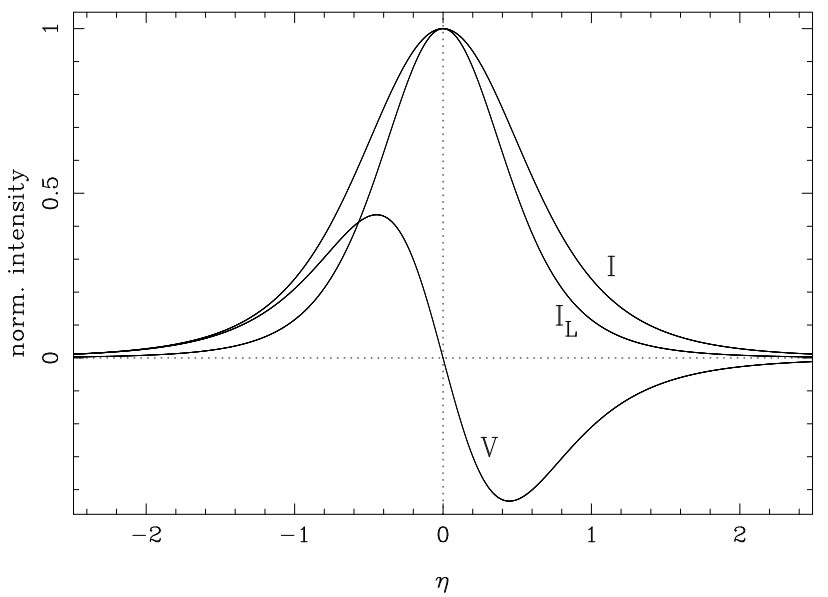

Fig. 3. - Polarization of synchrotron radiation from a collimated electron beam as function of re-scaled viewing angle $\eta=\gamma \sin \phi$. Given here are the Stokes parameters $I$ and $V$ and the linearly polarized flux $I_{L}=\sqrt{Q^{2}+U^{2}}$, normalized to $I(\eta=0) \equiv 1$.

an external observer are functions of $\gamma$ and the viewing angle $\phi$ between the line of sight and the plane of particle motion. For a collimated beam of mono-energetic electrons - i.e., all electrons have the same Lorentz factor $\gamma$ - moving in the $x y$ plane the circularly polarized flux (Stokes $V$ ) is

$$
V=-\frac{64 \eta}{7 \pi \sqrt{3}\left(1+\eta^{2}\right)^{3}}
$$

with $\eta=\gamma \sin \phi$. In this notation, the total intensity (Stokes $I$ ) of the radiation is given by

$$
I=\frac{7+12 \eta^{2}}{7\left(1+\eta^{2}\right)^{7 / 2}}
$$

(Michel 1991). The linearly polarized flux follows from Eqs. 39 and 40 via Eq. 23 in a straightforward manner. Notably, I normalized the expressions for $V$ and $I$ such that $I=1$ at $\phi=0$.

Isotropic electron motion. In most astrophysical plasmas, the electron velocities are distributed randomly and (more or less) isotropically. This implies that both right-handed and left-handed electron orbits contribute with equal probability, meaning the circular component of the radiation averages out: macroscopically, the synchrotron radiation becomes linearly polarized (though not perfectly; see the discussion below).

Taking into account the various projection effects, the degree of linear polarization is given by

$$
m_{L}=\frac{I_{\perp}-I_{\|}}{I_{\perp}+I_{\|}}
$$

with $I_{\perp}$ and $I_{\|}$denoting the intensities perpendicular and parallel to the magnetic field lines as projected 
onto the plane of the sky. Notably, the direction of polarization is perpendicular to the (projected) direction of the magnetic field. The actual value of $m_{L}$ depends on the spectrum of the synchrotron radiation which in turn is a function of the distribution of the electron energies. For an ensemble of mono-energetic electrons the result is $m_{L}=75 \%$ (Rybicki \& Lightman 1979). For a power law distribution of electron energies, the number of electrons $N$ varies with $\gamma$ like $N \propto \gamma^{-\Gamma}$, with $\Gamma$ being the energy index. In this case, the flux density of the synchrotron radiation $S_{\nu}$ varies with frequency $\nu$ like $S_{\nu} \propto \nu^{-\alpha}$, where $\Gamma=2 \alpha+1$. The degree of linear polarization is given by

$$
m_{L}=\frac{\Gamma+1}{\Gamma+7 / 3}
$$

(Ginzburg \& Syrovatskii 1965). For astrophysically realistic plasmas with $\alpha \approx 0 \ldots 1, m_{L} \approx 60 \ldots 80 \%$.

The relation given by Eq. 42 corresponds to a highly idealized situation, assuming optically thin plasmas, isotropic distributions of electrons, perfectly ordered homogeneous magnetic fields, and the absence of substantial perturbations. A major modification occurs for optically thick plasmas where each light ray experiences multiple scattering events. In those cases,

$$
m_{L}=\frac{1}{2 \Gamma+13 / 3}
$$

(Pacholczyk 1970); for $\alpha \approx 0, m_{L} \approx 16 \%$. Likewise, any disordering of the magnetic field leads to the polarization signal partially being averaged out, thus reducing $m_{L}$ well below the idealized theoretical values. In optically thick plasmas, the polarization is oriented parallel to the magnetic field component projected on the sky.

Yet another characteristic modulation of polarization is caused by shocks propagating through the plasma. The degree of linear polarization in a partially compressed plasma, compared to the case without compression, is reduced by a factor

$$
\mu=\frac{\delta}{2-\delta} \in[0,1] \text { with } \delta=\left(1-k^{2}\right) \cos ^{2} \epsilon
$$

where $0 \leq k \leq 1$ is the factor by which the length of the shocked region is reduced by compression, and $\epsilon$ is the angle between the line of sight and the plane of compression in the frame of reference of the emitter (Hughes, Aller, \& Aller 1985; Cawthorne \& Wardle 1988).

The amount of circular polarization follows roughly the relation $m_{C} \sim\left(\omega / \omega_{B}\right)^{-1 / 2}$ where $\omega$ is the (angular) frequency of the light and $\omega_{B}$ is the (angular) gyration frequency of the charged particle. As before, this relation assumes a uniform magnetic field and an isotropic distribution of particle velocities. In the extreme case
$\omega_{B} \gtrsim \omega$, the relation changes to $m_{C} \sim \omega / \omega_{B}$. In realistic astrophysical plasmas, $m_{C} \lesssim 1 \%$ (Angel 1974).

\subsection{Zeeman Effect}

Spectral emission or absorption lines experience modifications if the emitting or absorbing material is permeated by a magnetic field. For atomic or molecular transitions involving the orbital angular momentum only (i.e., no spin-orbit coupling), the quantum mechanical selection rules demand that the change of the magnetic quantum number $m$ has to obey $\Delta m \in[-1,0,1]$ - meaning there are three different transitions possible. As long as the atom is not exposed to external electric or magnetic fields, the three transitions are energetically degenerate; all three transitions have the same energy and cause spectral lines at the same frequency $\nu_{0}$. This changes in the presence of an external magnetic field as reported first by Zeeman (1897), hence Zeeman effect. As shown by a simple classical analysis (Rybicki \& Lightman 1979; Haken \& Wolf 1990), the magnetic field causes a splitting of the three initially degenerate energy levels. The spectral line splits into a set of three lines located at frequencies $\nu_{0}$ and $\nu_{ \pm}=\nu_{0} \pm \Delta \nu_{\mathrm{z}}$ for $\Delta m=0$ and $\Delta m= \pm 1$, respectively. The frequency offset is given by

$$
\Delta \nu_{\mathrm{z}}=\frac{1}{4 \pi} \frac{e}{m_{\mathrm{e}}} B=14 \mathrm{GHz} \times B
$$

where $e$ is the electric charge of the electron, $m_{\mathrm{e}}$ is the electron mass, and $B$ is the strength of the magnetic field in units of Tesla. The lines at $\nu_{-}, \nu_{0}$, and $\nu_{+}$ are denoted as $\sigma^{-}, \pi$ ("parallel"), and $\sigma^{+}$components, respectively.

The three spectral lines have distinct polarization properties that depend on the viewing geometry.

Transversal view. If the line of sight is perpendicular to the magnetic field lines, the observer notes three spectral lines corresponding to the $\pi$ and $\sigma^{ \pm}$components. All lines are linearly polarized. The polarization of the $\pi$ component is parallel to the magnetic field (hence the name), the polarizations of the $\sigma^{ \pm}$components are perpendicular to the field lines.

Longitudinal view. If the line of sight is parallel to the magnetic field lines, only the $\sigma^{ \pm}$components are visible. Both lines are circularly polarized, with $\sigma^{+}$ and $\sigma^{-}$being RHC and LHC, respectively. Notably, the orientation of polarization is defined relative to the direction of the magnetic field lines, not the direction of propagation of the light.

Assuming an angle $\theta$ between the magnetic field and the line of sight, the Stokes parameters resulting from the viewing geometry (e.g., Elitzur 2000) are

$$
\begin{aligned}
I & =I^{0} \sin ^{2} \theta \\
Q & =I^{0} \sin ^{2} \theta
\end{aligned}
$$




$$
\begin{aligned}
& U=0 \\
& V=0
\end{aligned}
$$

for the $\Delta m=0$ transition and

$$
\begin{aligned}
I & =\frac{1}{2} I^{ \pm}\left(1+\cos ^{2} \theta\right) \\
Q & =-\frac{1}{2} I^{ \pm} \sin ^{2} \theta \\
U & =0 \\
V & = \pm I^{ \pm} \cos \theta
\end{aligned}
$$

for the $\Delta m= \pm 1$ transitions, respectively. The $I^{0}, I^{ \pm}$ denote the maximum intensities of the respective lines, with $I^{+}\left(\nu_{+}\right)=I^{-}\left(\nu_{-}\right)=I^{0}\left(\nu_{0}\right)$.

As yet, I assumed strong Zeeman splitting that leads to distinct spectral lines, meaning intrinsic line widths $\Delta \nu \ll \Delta \nu_{\mathrm{z}}$. In astronomy, this is not always the case; the situation $\Delta \nu \gg \Delta \nu_{\mathrm{z}}$ is common. In the latter case, the total intensity as function of frequency, $I(\nu)$, exhibits a single line only. The Zeeman components are unveiled by analysis of the Stokes parameters as function of frequency, resulting in

$$
\begin{aligned}
I & =I^{0}+I^{+}+I^{-}=2 I^{0} \\
Q & =-\frac{\mathrm{d}^{2} I(\nu)}{\mathrm{d} \nu^{2}}\left(\Delta \nu_{\mathrm{z}} \sin \theta\right)^{2} \\
U & =0 \\
V & =\frac{\mathrm{d} I(\nu)}{\mathrm{d} \nu} \Delta \nu_{\mathrm{z}} \cos \theta
\end{aligned}
$$

Accordingly, $Q(\nu)$ and $V(\nu)$ are the scaled derivatives of $I(\nu)$. Observations especially of $V(\nu)$ are important examples of spectro-polarimetry.

As yet, I discussed the normal Zeeman effect occurring in atomic transitions without spin-orbit coupling. If spin-orbit coupling has to be taken into account, more complex patterns with multiple line components spaced non-equally occur. Details for this anomalous Zeeman effect depend on the total spins $S$, orbital angular momentum quantum numbers $L$, and total angular quantum numbers $J$. The $\pi$ and $\sigma^{ \pm}$components are given by $\Delta M=0$ and $\Delta M= \pm 1$ respectively, with $M$ denoting the total magnetic quantum number.

\subsection{Goldreich-Kylafis Effect}

We now revisit the Zeeman effect for the case of weak Zeeman splitting $\left(\Delta \nu \gg \Delta \nu_{\mathrm{z}}\right)$, meaning that the three Zeeman line components are not resolved. As indicated by Eq. 48, the linear polarization is rather weak in general (and zero at $\nu=\nu_{0}$ ). However, this result is based on the assumption that each transition $\Delta m=-1,0,1$ is equally likely.

In case that the transitions $\Delta m$ occur at different rates, increased linear polarization can occur (Goldre- ich \& Kylafis 1981, 1982). This is the case if the radiation field within the source is anisotropic: depending on the relative orientation of magnetic field and incident radiation, the amount of anisotropy, and the ratio of collisional and radiative excitation rates, the $\sigma^{ \pm}$and $\pi$ line components are excited with different probabilities. The radiative transition rates $\mathcal{T}$ for the $\sigma^{ \pm}$and $\pi$ components, which are proportional to the line intensities, are given by

$$
\begin{aligned}
& \mathcal{T}_{ \pm} \propto \frac{1}{2} \int \mathrm{d} \Omega\left[I_{\perp}+I_{||} \cos ^{2} \alpha\right] \\
& \mathcal{T}_{0} \propto \int \mathrm{d} \Omega I_{||} \sin ^{2} \alpha
\end{aligned}
$$

respectively, where $I_{\perp}$ is the incident light intensity polarized perpendicular to the magnetic field and the direction of propagation, $I_{\|}$is the intensity of the incident light polarized perpendicular to $I_{\perp}$ and the direction of propagation, $\Omega$ is the solid angle, and $\alpha$ is the angle between the travel path of the incident light and the magnetic field.

Depending on which transitions are excited preferentially, the resulting linear polarization is oriented either parallel or perpendicular to the (sky-projected) magnetic field lines. Under realistic conditions, one may expect degrees of linear polarization up to $\approx 10 \%$ in molecular lines from cool (temperatures $\$ 100 \mathrm{~K}$ ) interstellar matter at millimeter-radio wavelengths.

\subsection{Hanle Effect}

The Hanle effect (Hanle 1924) is a phenomenon that appears in fluorescent light. In the following, I assume a fluorescent gas permeated by a weak magnetic field $B$. The primary source of radiation is located in $x$ direction from the fluorescent gas, the line of sight as well as the magnetic field are directed along the $z$ axis. The primary light source emits radiation in $x$ direction with electric field components $E_{y, z}$. By geometry, the linear polarization component $E_{y}$ causes fluorescence observable in $z$ direction.

When regarding normal Zeeman splitting (Section $3.5)$ in the limit of a vanishing magnetic field $B \rightarrow 0$, one reaches the regime of coherent resonances. As long as the Zeeman splitting is on the order of the natural line width, i.e., $\Delta \nu_{z} \gtrsim \Delta \nu$, the $\sigma^{ \pm}$transitions are excited independently. The observer notes two circularly polarized waves with amplitudes $E_{+}$and $E_{-}$ and a combined intensity $I=E_{+}^{2}+E_{-}^{2}$. For vanishing magnetic fields, $\Delta \nu_{z} \ll \Delta \nu$ and both transitions $\sigma^{ \pm}$can be excited by the same photon (which is linearly polarized and thus can be decomposed in one RHC and one LHC component for exciting $\sigma^{+}$and $\sigma^{-}$, respectively). The observer notes fluorescent light with intensity $I=\left(E_{+}+E_{-}\right)^{2}$ and linear polarization 
$\left(m_{L}=100 \%\right.$ in ideal situations) directed along $y{ }^{*}$

Ideal coherence with maximum light intensity $I$ and maximum linear polarization occurs at $B=0$. For an increasing field strength $(|B|>0)$, the degree of coherence decreases, causing several effects: (i) the intensity of the fluorescent light decreases; (ii) the "de-phasing" of $\sigma^{+}$and $\sigma^{-}$causes (a) a depolarization, and (b) a turning of the plane of linear polarization by an angle $\beta$ such that $\tan \beta \approx \omega_{L} / \Gamma_{\text {line }}$, with $\omega_{L}$ being the Lamor frequency and $\Gamma_{\text {line }}$ being the effective (i.e., natural plus collisional) line width (e.g., Stenflo 1982).

\subsection{Interactions of Polarization and Matter}

\subsubsection{Partial Reflection}

A plane wave that falls onto the boundary between two homogeneous media " 1 " and " 2 " with indices of refraction $n_{1,2}-$ like, e.g., the boundary between two planeparallel plates made of different types of glass - is split into a reflected and a transmitted wave according to the law of reflection and Snell's law of diffraction, respectively. Except of the special case of normal incidence, reflectivity $R$ and transmissivity $T$ - the fractions of intensity being reflected and transmitted - are functions of linear polarization (Born \& Wolf 1999). Both parameters have to be re-written like

$$
\begin{aligned}
R & =R_{||} \cos ^{2} \alpha+R_{\perp} \sin ^{2} \alpha \\
T & =T_{||} \cos ^{2} \alpha+T_{\perp} \sin ^{2} \alpha
\end{aligned}
$$

with $\|$ and $\perp$ denoting linear polarizations parallel and perpendicular to the plane of incidence, respectively, and $\alpha$ being the angle between the electric field vector and the plane of incidence. Conservation of energy demands

$$
R+T=R_{\|}+T_{\|}=R_{\perp}+T_{\perp}=1 .
$$

All reflectivities and transmittivities depend on the ratio $n=n_{2} / n_{1}$ and the angle between the normal of the boundary and the incident light ray, $\theta$, via Fresnel's formulae. In general, $R_{\|} \neq R_{\perp}$ and $T_{\|} \neq T_{\perp}$; both, reflected and transmitted light become linearly polarized even if the incident light is unpolarized. In the specific case

$$
\tan \theta_{B}=n
$$

the component $R_{\|}$vanishes; $\theta_{B}$ is the Brewster angle. For the case of a transition from air to glass, $n \approx 1.5$ and $\theta_{B} \approx 57^{\circ}$. If the incident light is unpolarized, the reflected light is completely polarized; the transmitted light is polarized with a degree of linear polarization $m_{L} \approx 8 \%$.

\footnotetext{
* Similar situations can also occur at non-zero magnetic fields where magnetic term levels belonging to different angular momentum quantum numbers can cross. This is the base of levelcrossing spectroscopy.
}

\subsubsection{Birefringence}

Birefringence is a consequence of the electric anisotropy of crystals, meaning that the response of the medium to incident radiation (usually) depends on the direction of the electric field. This anisotropy is described by a symmetric dielectric tensor $\varepsilon$ that defines a system of principal dielectric axes with permittivities $\left\{\varepsilon_{x}, \varepsilon_{y}, \varepsilon_{z}\right\}$ (Fowles 1975; Huard 1997; Born \& Wolf 1999). In case of isotropic crystals, $\varepsilon_{x}=\varepsilon_{y}=\varepsilon_{z}$; this is the case for cubic crystals. In uniaxial crystals, $\varepsilon_{x}=\varepsilon_{y}$ while $\varepsilon_{y} \neq \varepsilon_{z}$; in biaxial crystals, $\varepsilon_{x} \neq \varepsilon_{y} \neq \varepsilon_{z}$. The two linear polarization components of a light ray - which are perpendicular to the direction of propagation - passing through an anisotropic crystal experience different permittivities and thus different indices of refraction $n \propto \sqrt{\varepsilon}$. The optic axes of a crystal correspond to light travel paths for which the two linear polarizations experience equal refraction. For the case of uniaxial crystals, the optic axis is the $z$ axis.

Birefringence is employed in various optical elements that modify the polarization state of light.

Polarizers. For a uniaxial crystal, the dielectric tensor is an ellipsoid. The index of refraction describes a circle in the $x y$ plane and ellipses in the $x z$ and $y z$ planes,with the optic axis corresponding to either the major or the minor axis of the ellipse depending on the material. For a wave polarized perpendicular to the optic axis, $E_{1}$, the propagation in the crystal is isotropic, the surfaces of equal phase in the plane of incidence are circular (regardless of the relative orientation of optic axis and plane of incidence). $E_{1}$ propagates through the crystal according to Snell's law; therefore it is referred to as ordinary wave. For a wave $E_{2}$ polarized perpendicular to $E_{1}$ (i.e., $E_{1} \perp E_{2}$ ), the components parallel and perpendicular to the optic axis experience different refractive indices and thus different phase velocities. The surfaces of equal phase in the plane of incidence are elliptical, rendering Snell's law invalid; accordingly, $E_{2}$ is referred to as extraordinary wave. Anisotropic crystals can be used to separate the ordinary and extraordinary waves, and thus the two linear polarizations, of unpolarized light. Various crystal geometries and combinations of crystals with different optic axis orientations - adding internal reflections - are used. The most common types of polarizers are polarizing beam splitters, Nicol prisms, and Glan-Thompson prisms.

Linear-to-circular converters. Let us consider a thin, plane-parallel plate cut from a uniaxial crystal located in the $x y$ plane, with the optic axis being the $y$ axis. In this case, the linear polarization components of a light wave propagating in $z$ direction experience two different refractive indices $n_{x, y}$. The corresponding phase velocities - the speeds of light within the crystal - are $c_{x}=c / n_{x}$ and $c_{y}=c / n_{y}$, respectively, with $c$ denoting the speed of light in vacuum. For light with wavelength $\lambda$ in vacuum, traveling a distance $d$ within the crystal, the two polarizations experience a phase 
shift

$$
\delta=\frac{2 \pi}{\lambda} d\left(n_{y}-n_{x}\right)
$$

When choosing $d$ such that $d\left(n_{y}-n_{x}\right)=\lambda / 4$, we find $\delta=\pi / 2$. Light with $\left\langle E_{x}^{2}\right\rangle=\left\langle E_{y}^{2}\right\rangle$, i.e., Stokes parameter $Q=0$ (cf., Eq. 22), becomes circularly polarized (cf., Eq. 4); for $Q \neq 0$, it becomes elliptically polarized. Optical elements with this property are referred to as quarter wave plates. For realistic uniaxial crystals with $\left|n_{y}-n_{x}\right| \approx 0.01 \ldots 0.1$ (Fowles 1975), $d \approx(3 \ldots 25) \lambda$, meaning that quarter wave plates are fragile devices.

Polarization plane turners. The relation provided by Eq. 53 has an additional consequence for linearly polarized light with components $E_{x, y}$. When choosing $d$ such that $d\left(n_{y}-n_{x}\right)=\lambda / 2$, the phase shift becomes $\delta=\pi$; the plate is a half wave plate. The component perpendicular to the optic axis, here $E_{x}$, is mirrored at the optic axis, i.e., $E_{x} \longrightarrow-E_{x}$ (cf., Eq. 3). If plane of polarization and optic axis are tilted by an angle $\beta$, the crystal turns the polarization plane of the light by an angle of $2 \beta$.

In a variety of materials, birefringence can be induced by external electric - Kerr effect - or magnetic - Cotton-Mouton effect - fields (Fowles 1975). This is employed in light modulators that need to be switched between different states at high speed. In case of the Kerr effect, the difference between the indices of refraction parallel $-n_{\|}-$and perpendicular $-n_{\perp}-$ to the orientation of an external electric field with amplitude $E$ is

$$
n_{\|}-n_{\perp}=K E^{2} \lambda
$$

where $\lambda$ is the wavelength of the light in vacuum and $K$ is Kerr's constant which is a function of the material. The Cotton-Mouton effect is the magnetic analogue of the (electric) Kerr effect; here the difference between the two indices of refraction is proportional to the squared strength of the external magnetic field.

A further variety is introduced by the Pockels effect observed in certain kinds of birefringent crystals upon application of external electric fields. Here the difference between the two indices of refraction is proportional to the electric field strength. This effect is used in Pockels cells that permit a rapid modulation of light. A common setup comprises a Pockels cell located between two static linear polarizers with perpendicular transmission axes. Via appropriate switching of the Pockels cell, it turns the plane of polarization of the infalling linearly polarized light, making the setup act as a very fast shutter (Fowles 1975).

\subsubsection{Faraday Rotation}

An external, static magnetic field $\mathbf{B}$ permeating a medium introduces an electric anisotropy. The impact on a light wave propagating through the medium is found by solving the equation of motion for an electron influenced by $\mathbf{B}$ and the oscillating electric field of the light wave $\mathbf{E}(t)$ (Fowles 1975; Rybicki \& Lightman 1979). From this, one finds a circular electric anisotropy with permittivities $\varepsilon_{R} \neq \varepsilon_{L}$, with $R$ and $L$ denoting right-hand and left-hand circular polarization, respectively. As any linearly polarized wave can be expressed as a superposition of one left-hand and one right-hand circularly polarized wave (Eq. 17), the electric anisotropy implies a characteristic rotation of the plane of polarization of linearly polarized light. The change of polarization angle can be expressed like

$$
\Delta \chi=\mathcal{V} B_{\|} l
$$

with $l$ denoting the length of the light travel path within the medium, $B_{\|}$being the magnetic field strength parallel to the light travel path, and $\mathcal{V}$ denoting Verdet's constant which is a function of wavelength and material (Fowles 1975).

In astrophysical situations, Faraday rotation occurs when light passes through magnetized interstellar plasma. This effect is quantified like

$$
\Delta \chi=\mathrm{RM} \times \lambda^{2}
$$

where $\lambda$ is the wavelength of the radiation (in the restframe of the medium) and RM is the rotation measure (in units of $\mathrm{rad} \mathrm{m}^{-2}$ )

$$
\mathrm{RM}=8.1 \times 10^{5} \int_{0}^{l} B_{\|} n_{e} \mathrm{~d} z
$$

with $B_{\|}$being the strength of the magnetic field (in units of Gauss) parallel to the line of sight (l.o.s.), $n_{e}$ being the electron number density (in $\mathrm{cm}^{-3}$ ), and $z$ being the coordinate (in parsec) directed along the l.o.s. (Rybicki \& Lightman 1979; Wilson, Rohlfs, \& Hüttemeister 2010).

\subsubsection{Faraday Depolarization}

In case of spatially inhomogeneous media, especially astrophysical plasmas, the Faraday effect can lead to a loss of linearly polarized intensity. If the rotation measure RM shows modulations with amplitudes $\triangle \mathrm{RM}$ on spatial scales smaller than the source, the source radiation experiences different Faraday rotation depending on the position. Observations that do not resolve the RM structure spatially superimpose waves with different orientations of their planes of linear polarization. This partially averages out the polarization signal, reducing the degree of linear polarization observed. A complete depolarization occurs when the medium is "Faraday thick"; from Eq. 56 one can estimate that this is the case if

$$
\Delta \mathrm{RM} \times \lambda^{2} \gg 1
$$


A more sophisticated calculation is possible when assuming that the RM fluctuations follow a Gaussian distribution with dispersion $\zeta \approx \Delta \mathrm{RM}$. For a source that is not resolved spatially by observations, one finds a depolarization law

$$
\xi=\exp \left(-2 \zeta^{2} \lambda^{4}\right)
$$

(Burn 1966; Tribble 1991). The parameter $\xi \in[0,1]$ is the ratio of observed and intrinsic degree of linear polarization.

\subsubsection{Polarization Conversion}

Under certain conditions, effects corresponding to those of birefringence in crystals can be observed also in astrophysical plasmas. In the following, I assume an electromagnetic wave with components $E_{x, y}$ propagating through a plasma in $z$ direction. The plasma is permeated by an ordered, static magnetic field directed along the $x$ axis. Plasma electrons accelerated by $E_{x}$ can move freely, whereas those accelerated by $E_{y}$ experience an additional magnetic Lorentz force - the response of the plasma to light becomes anisotropic, the plasma effectively becomes birefringent. In analogy to the relation given by Eq. 53, this effective birefringence introduces a phase shift between $E_{x}$ and $E_{y}$ that converts linear into circular polarization and vice versa; this effect is also referred to as Faraday conversion or Faraday pulsation (Pacholczyk \& Swihart 1970).

In relativistic astrophysical plasmas and at radio frequencies, one may expect to observe a certain level of circularly polarized light generated from initially linearly polarized radiation. Details depend strongly on the physical conditions within the plasma. Pacholczyk (1973) provides an estimate for the relation between the degrees of linear $\left(m_{L}\right)$ and circular $\left(m_{C}\right)$ polarization,

$$
\frac{m_{C}}{m_{L}} \propto n_{e} B_{\perp}^{2} \nu^{-3}
$$

where $n_{e}$ denotes the electron density, $B_{\perp}$ is the strength of the magnetic field component perpendicular to the line of sight, and $\nu$ is the observing frequency. In general, one may expect $m_{C} \lesssim 1 \%$.

\subsubsection{Chandrasekhar-Fermi Effect}

Linear polarization generated within a magnetized turbulent plasma - via, e.g., dust scattering (Section 3.1.2) or synchrotron radiation (Section 3.4) - is sensitive to the strengths of turbulence and magnetic field. The magnetic field is assumed to be "frozen" in the plasma. In case of weak fields, the field lines are dragged around by the turbulence, leading to a large r.m.s. dispersion in polarization angles. In case of strong fields, the field lines remain rather unimpressed by the turbulence, the dispersion in polarization angles is small.
Magnetic field, turbulence, and polarization angle are related (Chandrasekhar \& Fermi 1953) like

$$
B_{\perp}=\left(\frac{4}{3} \pi \rho\right)^{1 / 2} \frac{\sigma_{v}}{\sigma_{\chi}}
$$

where $B_{\perp}$ is the strength of the magnetic field perpendicular to the line of sight (in Gauss), $\rho$ is the mass density of the gas (in $\left.\mathrm{g} \mathrm{cm}^{-3}\right), \sigma_{v}$ is the r.m.s. velocity dispersion of the gas (in $\mathrm{cm} \mathrm{s}^{-1}$ ), and $\sigma_{\chi}$ is the dispersion of polarization angles (in radians).

\section{OBSERVATIONS}

Similar to the cases of photometry and spectroscopy, the techniques used for polarimetry of radiation from astronomical sources depend strongly on the energy of the light. In general, we can distinguish three different wavelength regimes. At radio wavelengths, we are able to record electromagnetic waves with their amplitudes and phases. At optical wavelengths, we are usually dealing with light intensity information. At $X / \gamma$-ray energies, a combination of high frequency and low flux usually implies that we observe and analyze individual photons. This being said, I note that the distinction can be blurred depending on the physical situation, and various techniques find application over a wide range of radiation energies.

\subsection{Sky Projection}

Our usual use of polarization parameters, especially of the Stokes parameters (Section 2.3.2), implicitly assumes that emitter and receiver of radiation are placed in a common, stationary system of coordinates. In astronomical observations, this is usually not the case: Earth rotation leads to a rotation of the field of view with respect to the observer. Assuming electric fields $E_{\mathrm{V}, \mathrm{H}}$ measured vertical and horizontal, respectively, with respect to the telescope, these are related to the Stokes parameters in the frame of reference of the source on sky like

$$
\begin{aligned}
& 2\left\langle E_{\mathrm{V}} E_{\mathrm{V}}^{*}\right\rangle=I+Q \cos 2 \psi+U \sin 2 \psi \\
& 2\left\langle E_{\mathrm{H}} E_{\mathrm{H}}^{*}\right\rangle=I-Q \cos 2 \psi-U \sin 2 \psi \\
& 2\left\langle E_{\mathrm{V}} E_{\mathrm{H}}^{*}\right\rangle=-Q \sin 2 \psi+U \cos 2 \psi+i V \\
& 2\left\langle E_{\mathrm{H}} E_{\mathrm{V}}^{*}\right\rangle=-Q \sin 2 \psi+U \cos 2 \psi-i V
\end{aligned}
$$

where $\psi$ denotes the parallactic angle counted from north to east and $i$ is the imaginary unit. Comparison to Eq. 27 shows that Earth rotation leads to a conversion from $Q$ to $U$ and vice versa from the point of view of the observer; $V$ remains unaffected (Thompson, Moran, \& Swenson 2004). The same result follows from Eq. 34 in a straightforward manner. 


\subsection{Terrestrial Atmosphere}

In most situations, the influence of Earth's atmosphere on polarization can be neglected; the atmosphere is neither birefringent nor dichroic. An important exception occurs at radio frequencies where the interaction of ionosphere and terrestrial magnetic field causes substantial Faraday rotation (Section 3.8.3). At an observing frequency $\nu=100 \mathrm{MHz}$, the angle of polarization is rotated by $\Delta \chi \approx 300^{\circ}$ at night to $\Delta \chi \approx 3000^{\circ}$ at daytime under typical atmospheric conditions; a reliable derivation of the true polarization angle is very difficult. As $\Delta \chi \propto \nu^{-2}$, this effect can be circumvented by selecting a sufficiently high observing frequency (Thompson, Moran, \& Swenson 2004; Clarke 2010).

An additional effect relevant mostly at optical wavelengths is the polarization of scattered sun or moon light. From the geometry argument presented in Section 3.1.1 it is straightforward to see that scattered light is linearly polarized. The degree of polarization reaches its maximum at an angular distance of $90^{\circ}$ from the light source, the polarization is oriented perpendicular to the line on sky connecting the source and the point observed. This behavior can be exploited for the calibration of polarimetric observations via dedicated observations of scattered light.

\subsection{Instrumental Polarization}

The design and geometry of a telescope inevitably influence the polarization of the collected light. Except of highly symmetric situations - most notably in Cassegrain focus telescopes - the (usually) multiple reflections within an optical system alter the polarization state of the light. The resulting instrumental polarization is given by the product of the Müller matrices of the individual telescope components as described in Section 2.3.3. These geometric effects need to be corrected in the course of data analysis and/or by dedicated corrective optics in the telescope (see, e.g., Thum et al. 2008 for a discussion of Nasmyth optics).

Even though one may correct for the influence of the telescope geometry, realistic instruments are not perfect. In the most general case, the observed Stokes parameter values deviate from the actual ones by

$$
\Delta \mathbf{S}=-\frac{1}{2}\left[\begin{array}{rrrr}
\gamma_{++} & \gamma_{+-} & \delta_{+-} & -i \delta_{-+} \\
\gamma_{+-} & \gamma_{++} & \delta_{++} & -i \delta_{--} \\
\delta_{+-} & -\delta_{++} & \gamma_{++} & i \gamma_{--} \\
-i \delta_{-+} & i \delta_{--} & -i \gamma_{--} & \gamma_{++}
\end{array}\right] \mathbf{S}
$$

where $\mathbf{S}$ is the Stokes vector of the infalling light, the $\gamma_{\mathrm{xx}} \ll 1$ are error terms related to the gains, or efficiencies, of the optical paths for the $E_{x, y}$ components, and the $\delta_{\mathrm{xx}} \ll 1$ are error terms related to the leakage, also known as cross-talk, meaning the mutual influence of the optical paths for separate polarizations; ${ }^{\dagger}$ the (maximum) number of error terms is seven (Sault, Hamaker,
\& Bregman 1996). The expression given by Eq. 63 assumes that Stokes parameters are derived from linear polarization components via Eq. 27. If Stokes parameters are derived from circular polarization components (Eq. 29), Eq. 63 can be applied to a Stokes vector with $Q, U$, and $V$ being interchanged with $V, Q$, and $U$, respectively (cf., Eq. 27 vs. Eq. 29).

The actual calibration procedure depends strongly on the telescope(s) used. In general, calibration involves observations of one or more unpolarized astronomical reference sources - probing interactions between $I$ on the one hand and $Q, U$, and $V$ on the other hand - and observations of one or more polarized calibration sources, possibly several times at different parallactic angles - probing interactions between $Q, U$, and $V$ by comparison of observed and expected values (e.g., Clarke 2010).

\subsection{Polarization Statistics}

Whereas the effects discussed in Section 4.1-4.3 introduce systematic errors into polarization data, we now discuss the statistical uncertainties and limits to be taken into account. First of all, it is important to note that, in general, polarimetric observations require much better signal-to-noise ratios $(S / N)$ than photometric ones. As the degrees of linear or circular polarization $m_{L, C} \leq 1$ are usually much smaller than unity, the signal-to-noise ratio $(S / N)_{I}$ of the total intensity (Stokes $I$ ) signal can be related to the $S / N$ of the polarized intensity $(S / N)_{P}$ like $(S / N)_{P} \approx m_{L, C}(S / N)_{I}$. $^{\ddagger}$ This implies that a detection of a weak polarization signal may require very high $(S / N)_{I}$.

In case of linear polarization, statistical measurement uncertainties lead to a bias in the measured values for $m_{L}$. This is due to $m_{L}$ being positive definite by construction (Eq. 25): even if $Q$ and $U$ are symmetric random variables centered at zero, the sum of their squares is not; the values of $m_{L}$ follow a Rice distribution. A de-biasing can be attempted by subtracting from each of $Q$ and $U$ the corresponding statistical uncertainty in squares before the calculation of $m_{L}$. For high $(S / N)_{P}$, the statistical errors of $m_{L}, \sigma_{m}$, and polarization angle $\chi, \sigma_{\chi}$, are related like $\sigma_{\chi}=\sigma_{m} /\left(2 m_{L}\right)$ (in units of radians); the values of $\chi$ follow a normal distribution. For low $(S / N)_{P}$, the values for $Q$ and $U$ scatter around the origin of the $Q U$ plane, the distribution of the $\chi$ values becomes more and more platykurtic for lower and lower $(S / N)_{P}($ Clarke 2010).

of the order of few per cent - i.e., the same order as the actual polarization signal in many cases.

$\ddagger$ This relation is strictly valid only when the polarization is derived from sums or differences of intensities, especially in optical polarimetry. In cases where the polarization is derived from multiplications of fields or from correlations, the process of multiplication leads to non-Gaussian error distributions, modifying the noise estimates by factors of several.

\footnotetext{
${ }^{\dagger}$ Even though the $\delta$ and $\gamma$ terms are small, they can easily be
} 


\subsection{Radio Observations}

At radio wavelengths, the infalling radiation can be recorded and analyzed as waves with full amplitude and phase information; due to fundamental quantum limits, this is possible at frequencies up to about one $\mathrm{THz}$ (Thompson, Moran, \& Swenson 2004; Wilson, Rohlfs, \& Hüttemeister 2010). Regardless of the actual design details, a radio telescope can be modeled as a cross of two dipoles aligned along the $x$ and $y$ axes, respectively. We may assume, as usual, light propagating along the $z$ direction with linear polarization components $E_{x, y}$. Each of the two dipoles receives the corresponding polarization component and converts it into an electric voltage that can be recorded and processed electronically - radio receivers are polarimeters by construction ${ }^{\S}$ (see also Hamaker, Bregman, \& Sault 1996; Hamaker \& Bregman 1996; Sault, Hamaker, \& Bregman 1996; Hamaker 2000, 2006 for an exhaustive discussion). The signals received by the dipoles can be autocorrelated - resulting in the time-averaged products $\left\langle E_{x} E_{x}^{*}\right\rangle$ and $\left\langle E_{y} E_{y}^{*}\right\rangle$ - as well as crosscorrelated resulting in $\left\langle E_{x} E_{y}^{*}\right\rangle$ and $\left\langle E_{y} E_{x}^{*}\right\rangle$ (using complex exponential notation). The Stokes parameters $I, Q, U, V$ are derived from these products via Eq. 27 in a straightforward manner. A receiving system sensitive to both polarizations $E_{x, y}$ (or $E_{R, L}$ for circular polarization) is referred to as a dual-polarization receiver.

The cross of dipoles also serves as a model for radio receivers sensitive to circular polarization. For this we assume that (i) the signals from the two dipoles are sent to a common electronic processor and summed up coherently and (ii) a phase shift of $\pm \pi / 2$ is applied to the signal from the $y$ dipole. Accordingly, the two voltages will be in phase and trigger a signal if the infalling light is either RHC or LHC polarized, depending on the sign of the phase shift. The receiver is a singlepolarization receiver sensitive to either RHC or LHC; it can be extended to a dual-polarization receiver by adding a second cross of dipoles with opposite phase shift. By symmetry, these arguments hold also for the case of sending waves for radar astronomy; here, usually circularly polarized radio light is used (Ostro 1993). Due to the technical simplicity of radio polarimetry, recent efforts have been directed toward simultaneous multi-wavelength polarimetry (cf., e.g., K.-T. Kim et al. 2011; Lee et al. 2011) aimed at the measurement of differential parameters like dispersion measures.

The choice of polarization is important when combining the signals from two antennas located at large distance, e.g., in Very Long Baseline Interferometry (VLBI). The discussion provided in Section 4.1 also implies that the raw observed values for $Q$ and $U$ are functions of the geographic positions when using linear polarization receivers. This problem is circumvented

$\S$ This excludes radio techniques sensitive to total intensities only, notably bolometers. These have to be treated like optical telescopes (Section 4.6).
$I\left(0^{\circ}\right)+I\left(90^{\circ}\right)$
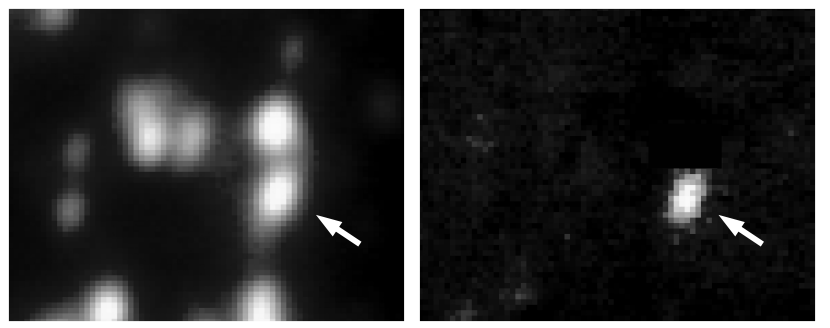

Stokes $I$

Stokes $Q$

Fig. 4. - Polarimetric imaging of Sagittarius A* (Sgr A*), the supermassive black hole at the center of the Milky Way, at $2.2 \mu \mathrm{m}$. Sgr $\mathrm{A}^{*}$ is indicated by an arrow, the surrounding sources are stars; the angular resolution is $\approx 50$ mas. The difference between the synchrotron source Sgr A* and the stars emitting thermal radiation becomes evident in the Stokes $Q$ image; at the time of observation, $m_{L} \approx 20 \%$ (Trippe et al. 2007).

by using circular polarization receivers which are insensitive to Earth rotation.

\subsection{Optical Observations}

At optical wavelengths, polarimetry is limited to light intensities rather than electric waves. Polarimetric measurements require the use of polarizers plus auxiliary optical elements placed in the optical path before the detector (usually a CCD array; e.g., Tinbergen 1996).

Linear polarization can be probed by measuring the intensity of the received light, $I(\psi)$, polarized at a parallactic angle $\psi$ to the $x$ (north-south) axis of the usual $x y$ coordinate system of the Stokes parameters (Section 2.3.2). The Stokes parameters $Q$ and $U$ are related to these intensities like

$$
\begin{aligned}
\frac{Q}{I} & =\frac{I\left(0^{\circ}\right)-I\left(90^{\circ}\right)}{I\left(0^{\circ}\right)+I\left(90^{\circ}\right)} \\
\frac{U}{I} & =\frac{I\left(45^{\circ}\right)-I\left(135^{\circ}\right)}{I\left(45^{\circ}\right)+I\left(135^{\circ}\right)}
\end{aligned}
$$

with $I \equiv I\left(0^{\circ}\right)+I\left(90^{\circ}\right) \equiv I\left(45^{\circ}\right)+I\left(135^{\circ}\right)$ being Stokes $I$ as usual (Kitchin 2009; Witzel et al. 2011); see Fig. 4 for an example. Alternatively, one may measure $I(\psi)$ at multiple - at least four - values of $\psi$ and model the measurement values with the function

$$
q(\psi)=\frac{I(\psi)-I\left(\psi+90^{\circ}\right)}{I(\psi)+I\left(\psi+90^{\circ}\right)}=m_{L} \cos [2(\psi-\chi)]
$$

with $m_{L}$ denoting the degree of linear polarization and $\chi$ denoting the polarization angle as defined in Section 
2.1 .5 (e.g., Ott, Eckart \& Genzel 1999; Trippe et al. 2010). Using Eq. 65 with a sufficiently large number of measurement values $(\geq 8)$ with a good sampling of $\psi$ values helps to recognize instrumental polarization effects in the data. "

In order to filter $I(\psi)$ out of the infalling radiation, various types of polarizers can be used. One possibility are wire-grid polarizers (Huard 1997) that pass light polarized perpendicular to the grid and reflect the other component. For each value of $\psi$, the grid is rotated into the required position and an image of the target is taken (e.g., Ott, Eckart \& Genzel 1999). A more efficient approach is provided by using a combination of (i) a Wollaston prism that splits the infalling light into ordinary and extraordinary linearly polarized rays, and (ii) a half wave plate (HWP) that permits turning the plane of linear polarization (cf., Section 3.8.2). A complete measurement cycle involves taking two images, each showing the ordinary and extraordinary ray images of the target: one with the HWP turned to a position corresponding to $\psi=0^{\circ} / 90^{\circ}$, one with the HWP turned such that $\psi=45^{\circ} / 135^{\circ}$ is observed. The linear polarization of the target is then derived via Eq. 64 in a straightforward manner (e.g., Witzel et al. 2011).

An analysis of circular polarization requires the use of a quarter wave plate (QWP). The QWP converts circular into linear polarization; the linearly polarized light can be analyzed as discussed above. For a QWP with its axis of minimum index of refraction - its fast axis - being the $x$ axis in our usual (Section 2.1) coordinate system, its impact on circularly polarized light can be written in Jones calculus like

$$
\left[\begin{array}{ll}
1 & 0 \\
0 & i
\end{array}\right]\left[\begin{array}{r}
1 \\
\mp i
\end{array}\right]=\left[\begin{array}{r}
1 \\
\pm 1
\end{array}\right]
$$

which denotes the application of the Jones matrix of the QWP to a circularly (RHC or LHC) polarized wave, resulting in a linearly polarized wave with diagonal plane of polarization (Fowles 1975). Comparison of the result to the definition of the Stokes parameters (Section 2.3.2) shows that the QWP converts $V$ to $U$. Accordingly, we can now derive $V$ from an analysis of linear polarization according to Eq. 64, resulting in

$$
\frac{V}{I}=\frac{I\left(45^{\circ}\right)-I\left(135^{\circ}\right)}{I\left(45^{\circ}\right)+I\left(135^{\circ}\right)}
$$

(cf., e.g., Goodrich, Cohen \& Putney 1995). I note that the choice of QWP orientation - here along the $x$ axis

\footnotetext{
This is straightforward to see in the special case of Cassegrain focus observations. In this case, the target polarization is fixed with respect to the sky whereas the instrumental polarization is fixed with respect to the telescope. We may obtain observations at two (or more) different hour angles and model each data set as a superposition of two cosine profiles as defined in Eq. 65: one corresponding to the target polarization and one corresponding to the instrumental polarization. A polarization signal which remains unchanged - in sky coordinates - at different hour angles is intrinsic to the target.
}

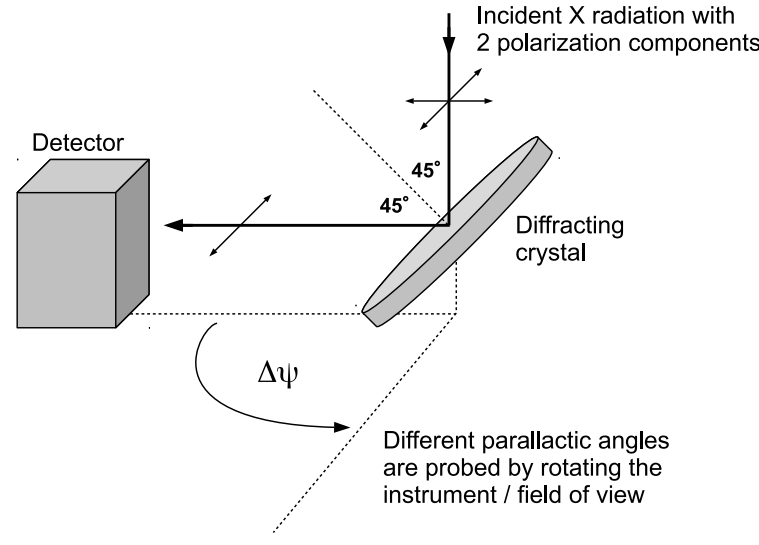

Fig. 5.- Illustration of Bragg diffraction polarimetry.

- is arbitrary; for example, a diagonal orientation of the fast axis leads to a conversion from $V$ to $Q$ (e.g., Fowles 1975).

\section{7 $\quad$ X and $\gamma$ Ray Observations}

Due to the high photon energies and short wavelengths involved, optical elements used at optical wavelengths become transparent at $X / \gamma$ ray energies. Polarimetry at these wavelengths can be based on any of three distinct physical effects.

Bragg diffraction. Light with sufficiently short (less than a few nanometers) wavelength $\lambda$ falling onto a crystal is reflected by the crystal according to Bragg's law

$$
n \lambda=2 d \sin \theta \quad ; \quad n=1,2,3, \ldots
$$

with $d$ denoting the distance between two consecutive atomic layers measured perpendicular to the surface of the crystal, $\theta$ denoting the angle of incidence measured between the infalling light ray and the surface of the crystal, and $n$ being the order of diffraction (Born \& Wolf 1999). Using the relation derived in Section 3.1.1 it becomes evident that the reflected light is linearly polarized, with the component parallel to the surface prevailing. If incident and reflected rays are perpendicular - meaning $\theta=45^{\circ}$ - only the polarization component parallel to the surface of the crystal remains in the reflected light. The linear polarization state of a science target can be derived by rotating the field of view of the instrument and observing the resulting cosinusoidal profile equivalent to Eq. 65; I illustrate the measurement geometry in Fig. 5. As Bragg's law is strictly valid only for one specific wavelength, this method is, a priori, limited to very narrow energy bands at each order of diffraction. This condition can be relaxed by using "mosaicked" crystals composed of many small crystalets, thus providing a range of $d$ values for diffraction (Silver \& Schnopper 2010). 
Bragg diffraction polarimetry was the method used for the only X-ray polarimeter ever implemented in a space telescope, the OSO-8 satellite. It was used to measure the polarization of the Crab nebula (Weisskopf et al. 1978), resulting in the "first and only highprecision X-ray polarization measurement obtained for any cosmic source" (Silver \& Schnopper 2010).

Scattering polarimetry. As discussed in Section 3.1.1, Thomson or Compton scattering of photons by electrons is sensitive to the linear polarization of the incident photons. This is exploited in scattering polarimeters. We may assume, as usual, partially polarized light composed of photons propagating in $z$ direction. These photons arrive at a scattering detector that provides material for scattering the incident light; the scattering detector is located at the origin of the $x y$ plane. A certain fraction of the arriving photons will be scattered at right angles into the $x y$ plane where they are recorded by a calorimeter. In case of no polarization, the distribution of scattered photons in the $x y$ plane will be isotropic. If the light is partially linearly polarized, photons will be scattered preferentially perpendicular to the direction of polarization projected onto the $x y$ plane; the resulting distribution is given by expressions equivalent to Eq. 65 when taking into account the instrument and scattering geometries (McConnell 2010).

Photoelectron tracking. Irradiation of $\mathrm{X}$ rays on a medium can cause the release of photoelectrons. The direction of photoelectron emission is a function of the polarization of the incident light. Assuming a linearly polarized $\mathrm{X}$ ray photon propagating in $z$ direction that causes the emission of a photoelectron at the origin of the $x y$ plane, the differential cross section of photoelectron emission is given by

$$
\frac{\mathrm{d} \sigma}{\mathrm{d} \Omega} \propto \frac{\sin ^{2} \theta \cos ^{2} \phi}{[1-\beta \cos \theta]^{4}}
$$

where $\sigma$ is the cross section, $\Omega$ denotes the solid angle, $\beta$ is the electron speed in units of speed of light, $\theta$ is the angle between the path of the incident photon and the path of the emitted photoelectron, and $\phi$ is the angle between the path of the photoelectron and the direction of polarization of the photon projected onto the $x y$ plane (Bellazzini \& Spandre 2010). Accordingly, the distribution of photoelectrons is a function of photon polarization, resulting in a characteristic $\cos ^{2} \phi$ pattern in the $x y$ plane.

Compared to classical Thomson scattering, the photoelectric effect is more efficient in analyzing the photon polarization: whereas the differential cross section of Thomson scattering decreases with $\theta$ increasing from $0^{\circ}$ to $90^{\circ}$ (cf., e.g., Rybicki \& Lightman 1979), it strongly increases in case of photoelectron emission (Eq. 69). At energies of few $\mathrm{keV}$ (i.e., $\beta \lesssim 0.1$ ), the differential cross section peaks at $\theta \approx 90^{\circ}$, meaning most of the photoelectrons are emitted within or close to the $x y$ plane. The electron paths can be traced by semi- conductor (CCD), gas, or scintillation photo-detectors located in the $x y$ plane; again, the degree and orientation of macroscopic polarization can be derived from the photoelectron distribution via Eq. 65 (or equivalent expressions).

The use of a certain method for $\mathrm{X} / \gamma$ ray polarimetry is largely dictated by the photon energies. Soft $\mathrm{X}$ rays can be analyzed with either method; the analysis of hard $\mathrm{X}$ and $\gamma$ rays is usually limited to Compton scattering polarimetry (cf., e.g., Bloser et al. 2010).

\section{SCIENCE CASES}

\subsection{Solar and Stellar Physics}

Across the Hertzsprung-Russell diagram, stars, including the sun, are known to posess magnetic fields with field strengths ranging from a few to several ten thousand Gauss (e.g., Schrijver \& Zwaan 2000; Berdyugina 2009). In case of hot stars with radiative outer layers (roughly, spectral classes $\mathrm{O}-\mathrm{A}$ ), magnetic fields are supposed to be "fossil", i.e., inherited from the intergalactic medium the stars formed from; in case of stars with convective outer layers (approximately spectral classes $\mathrm{F}-\mathrm{M}$ ), magnetic fields are generated by dynamo processes (e.g., Berdyugina 2009). Accordingly, analyses of stellar magnetic fields are able to constrain solar and stellar dynamo models.

Stellar magnetic fields - including here also magnetic white dwarfs with $B \lesssim 10^{9} \mathrm{G}$ (Jordan 2009) - can be analyzed via spectropolarimetry of absorption lines that are affected by Zeeman splitting (Section 3.5). According to Eqs. 46, 47 and 48, the orientation of the field lines can be assessed from the relative strength of linear and circular polarization (see, e.g., Donati \& Landstreet 2009 for a review). In case of weak Zeeman splitting (Eq. 48) - a common case in stellar spectral lines - the magnetic field strength $B$ enters (via Eq. 45) linearly into $V$ and quadratically into $Q$. On the one hand, this makes it possible to estimate $B$ directly from the $V(\nu)$ profile; on the other hand, this complicates the analysis of the field orientation. Spatially resolved maps of magnetic fields of the sun (e.g., Stenflo 2013) or stars (e.g., Arzoumanian et al. 2011), usually based on circular polarization, are referred to as magnetograms.

Complementary to Zeeman effect measurements, the Hanle effect (Section 3.7) can be used to probe the magnetic field of the sun (Berdyugina 2004; Milić \& Faurobert 2012). This is achieved by simultaneous spectropolarimetric observations of several molecular fluorescent lines; important diagnostic molecules are $\mathrm{C}_{2}$ and $\mathrm{MgH}$. In addition, scattering polarization by Rayleigh and Raman scattering probes the physical conditions in stellar atmospheres (e.g., Sampoorna, Nagendra, \& Stenflo 2013). 


\subsection{Planetary System Bodies}

\subsubsection{Solid Surfaces}

Sunlight reflected at a solid surface - like the ones of rocky planets or asteroids - becomes partially linearly polarized due to scattering polarization (Section 3.1). Unsurprisingly, the observed degree of polarization is a function of the relative position of observer, reflecting body, and the star (the phase angle in case of the solar system). The maximum degree of linear polarization, in the following denoted with $\bar{m}_{L}$, is a function of wavelength and of the structure of the reflecting material. The interplay between absorption and scattering of light causes the Umov effect, a characteristic anticorrelation between $\bar{m}_{L}$ and geometric albedo $\mathcal{A}$ of a solid surface (Bowell \& Zellner 1974). For a given material - like lunar regolith, sand, basalt, or granite polarization and albedo are related like

$$
\log (\mathcal{A})=-c_{1} \log \left(\bar{m}_{L}\right)+c_{2}
$$

at visible wavelengths, with constants $c_{1} \approx 1$ and $c_{2} \approx-2$ (for $\left.\mathcal{A}, m_{L} \in[0.01,1]\right)$. Notably, this implies degrees of polarization close to $100 \%$ for very low albedos. Observed deviations from this relation indicate a change in the structure of the surface material; accordingly, $\mathcal{A}-\bar{m}_{L}$ diagrams can be used to assess the surface composition of a planet or any other solid body. In addition, characteristic variations of $\bar{m}_{L}$ with time can be used to estimate the rotation period and/or surface profile of a small body (e.g., an asteroid) that is not resolved spatially by observations (e.g., Ishiguro et al. 1997; Cellino et al. 2005).

Radar astronomical observations (Ostro 1993; Campbell 2002) exploit the polarization state of the reflected radio light. The transmitted radar signal has a well defined polarization state (usually 100\% circular). In case of a single reflection at an ideal dielectric surface, the circular polarization state of the echo signal is inverted with respect to the transmitted signal, the linear polarization state (expressed via Stokes $Q$ by proper choice of coordinates) remains unchanged (cf., Eq. 33). Multiple scattering and/or refraction at rough surfaces lead to some of the echo light being in the same circular polarization state and/or inverted linear polarization state compared to the infalling light. Denoting the polarization states as the "same" (S) and "opposite" (O) ones with respect to the transmitted radar signal, one can define the polarization ratios

$$
\mathcal{R}_{C}=\frac{\Sigma_{\mathrm{SC}}}{\Sigma_{\mathrm{OC}}} \quad \text { and } \quad \mathcal{R}_{L}=\frac{\Sigma_{\mathrm{OL}}}{\Sigma_{\mathrm{SL}}}
$$

with "L" and "C" referring to linear and circular polarization, respectively, and $\Sigma$ denoting the radar cross section of the target. Accordingly, both $\mathcal{R}_{L}$ and $\mathcal{R}_{C}$ would be zero for an ideal smooth surface. For most solar system objects, $\mathcal{R}_{C} \lesssim 0.3$, with the notable exception of the icy moons of Jupiter for which $\mathcal{R}_{C} \gtrsim 1$
(Ostro 1993).

\subsubsection{Atmospheres}

Reflection of light at (sufficiently dense) planetary atmospheres (e.g., Buenzli \& Schmid 2009, and references therein) is mainly affected by two (linearly) polarizing processes: (i) Rayleigh scattering at molecules and aerosol haze particles, and (ii) refraction and reflection at liquid droplets in clouds. Whereas individual interactions can lead to degrees of linear polarization up to $100 \%$, the signal observed by a distant observer is the average over multiple light rays, which partially averages out the polarization signal and reduces the observed degree of polarization. The actual polarization levels depend strongly on the reflection geometry and the chemical composition of the atmosphere. Within and around the regime of visible wavelengths, observed levels of polarization - integrated over the planetary disks - are $<5 \%$ for Venus, 5-10\% for Jupiter and Saturn, and up to $\approx 50 \%$ for Titan (Saturn's moon).

Quantitative investigations of the polarization properties of planetary atmospheres require numerical modeling (e.g., Buenzli \& Schmid 2009). The polarization of - intrinsically unpolarized - starlight reflected from planets is used for direct imaging of exoplanets via polarimetric differential imaging (e.g., Milli et al. 2013).

\subsection{Interstellar Matter}

Interstellar space is filled with diffuse matter occurring in a large variety of states, from cold dense molecular (main species being $\mathrm{H}_{2}, \mathrm{CO}$, and $\mathrm{H}_{2} \mathrm{O}$ ) clouds with temperatures $T$ of few Kelvin and (hydrogen) particle densities $10^{3 \ldots 5} \mathrm{~cm}^{-3}$ up to the hot ionized (coronal) medium (main species being $\mathrm{H}$ II, C IV, N V, and $\mathrm{O}$ VI) with $T \approx 10^{6} \mathrm{~K}$ and hydrogen densities $\approx 3 \times 10^{-3} \mathrm{~cm}^{-3}$. In addition, interstellar dust is omnipresent throughout galaxies (see, e.g., Kwok 2007 for a detailed overview).

The interplay of interstellar dust and galactic magnetic fields (Sections 3.1.2 and 5.8) is responsible for the interstellar polarization of scattered starlight (see, e.g., Das, Voshchinnikov, \& Il'in 2010; Matsumura et al. 2011 for recent discussions); the degree of linear polarization is approximately given by Serkowski's law and, accordingly, ranges from a few to about ten per cent (Draine 2003). In case of circumstellar material in the immediate vicinity of a star, scattering polarization can arise from:

(i) The alignment of dust grains in the magnetic field of a circumstellar disk or star-forming nebula.

(ii) Scattering at spherical or randomly oriented dust grains; in this case, polarization arises from geometry because the incident light arrives from a well-defined direction - the star.

(iii) Scattering at magnetically aligned dust grains plus dichroic absorption by foreground material, 
leading (also) to circular polarization with $m_{C} \lesssim$ $20 \%$ (Kwon et al. 2013).

In case (i), infrared polarimetric imaging has revealed the magnetic field structures in disks around young stars as well as characteristic "hour-glass" field geometries in star forming regions (e.g., Cho \& Lazarian 2007; Sugitani et al. 2010). In case (ii), polarimetric imaging of circumstellar material shows a highly symmetric circular pattern centered at the star, with the orientation of polarization being perpendicular to the direction of the incident radiation. This has been used to analyze (proto)stars embedded in dense interstellar matter (e.g., Saito et al. 2009). Circular scattering/absorption polarization (case iii) has been observed only in a few star forming regions (Kwon et al. 2013).

\subsection{Astrobiology}

Complex helical organic molecules in terrestrial life forms - like amino acids - show homochirality: out of two helix orientations possible, only one is used exclusively. This phenomenon implies that one of the two orientations was preferred in pre-biotic chemistry. A possible cause is circularly polarized light in star forming regions (Section 5.3) leading to preferential photodissociation of organic molecules with one specific orientation. This causes an excess of molecules with a given orientation and, eventually, on Earth to which organic matter is transported via comets and meteoroids (De Marcellus et al. 2011; Kwon et al. 2013).

Homochirality causes light reflected from certain biological surfaces to be circularly polarized (cf., Section 3.3). This effect can - in principle - be exploited for detecting life on other planets via (spectro)polarimetry of starlight reflected from the surface (e.g., Sparks et al. 2012).

\subsection{Astronomical Masers}

Stimulated emission of radiation at radio frequencies maser radiation - can be observed from the interstellar matter in star-forming regions and from the circumstellar envelopes of late-type (super)giant stars (e.g., Kwon \& Suh 2012). Maser radiation is emitted as molecular line emission with very high brightness temperatures up to roughly $10^{12} \mathrm{~K}$. Species known to act as astrophysical maser media are the molecules $\mathrm{OH}, \mathrm{H}_{2} \mathrm{O}$, $\mathrm{CH}_{3} \mathrm{OH}, \mathrm{NH}_{3}, \mathrm{HC}_{3} \mathrm{~N}, \mathrm{H}_{2} \mathrm{CO}, \mathrm{CH}, \mathrm{SiO}, \mathrm{SiS}$, and $\mathrm{HCN}$, plus atomic hydrogen $(\mathrm{H})$. Maser radiation has been observed at frequencies from $1.61 \mathrm{GHz}$ (from $\mathrm{OH}$ ) to $662.4 \mathrm{GHz}$ (from $\mathrm{H}$ ), i.e., across the entire radio regime (Reid \& Moran 1981; Elitzur 1982; Townes 1997).

Astronomical masers tend to show substantial Zeeman line splitting caused by magnetic fields permeating the maser medium. In the case of strong $\left(\Delta \nu_{\mathrm{z}} \gg \Delta \nu\right)$ Zeeman splitting, the resulting line polarizations are given by Eqs. 46 and 47 . In case of weak Zeeman splitting $\left(\Delta \nu_{\mathrm{z}} \ll \Delta \nu\right)$ however we have to take into account that maser radiation is caused by stimulated, coherent emission, meaning a coherent superposition of electric waves. For a single electromagnetic wave, the resulting Stokes parameters, in units of Stokes $I$, are

$$
\begin{aligned}
& \frac{Q}{I}=-1+\frac{2}{3 \sin ^{2} \theta} \\
& \frac{U}{I}= \pm \frac{2}{3 \sin ^{2} \theta}\left(3 \sin ^{2} \theta-1\right)^{1 / 2} \\
& \frac{V}{I}=0
\end{aligned}
$$

with $\theta$ denoting the angle between the magnetic field and the line of sight (Elitzur 1991, 2000). For $\sin ^{2} \theta \leq$ $1 / 3$ (i.e., $\theta \lesssim 35^{\circ}$ ), $Q / I=1$ and $U / I=0$. As $(Q / I)^{2}+(U / I)^{2}=1$ for all $\theta$, a wave emitted by an ideal maser is always fully linearly polarized. When averaging over multiple waves - as in any realistic astronomical observation - the sign ambiguity in $U / I$ causes Stokes $U$ to average out; only $Q$ remains, implying a partial linear polarization with $m_{L}=Q / I$. However, more recent calculations based on numerical simulations of realistic maser radiation fields find that the analytical estimates quoted above suffer from over-simplifications; the actual levels of linear polarization should be substantially smaller than the ones predicted by Eq. 72 (Dinh-V-Trung 2009). Furthermore, already moderate $\left(\Delta \nu_{z}<\Delta \nu\right)$ Zeeman splitting introduces circular polarization with amplitudes as high as $m_{C} \approx 20 \%$, with frequency-dependent profiles $V(\nu)$ similar to Eq. 48 for sufficiently small $\theta \lesssim 30^{\circ}$ (Elitzur 2000; Dinh-V-Trung 2009).

\subsection{Pulsars}

Pulsars are neutron stars with strong magnetospheres. Their radiation is composed of thermal radiation from the neutron star surface - at temperatures $T \approx 10^{6} \mathrm{~K}$ - and, predominantly, non-thermal synchrotron and curvature radiation created within the stellar magnetosphere. The observational pulsar phenomenology is given by geometry: the magnetic axis of the star is tilted relative to its spin axis. If the magnetic axis points to the observer during a rotation period, the radiation from the magnetosphere becomes visible as a short pulse of light. Observed pulse periods are located roughly in the range from few milliseconds to tens of seconds, with most pulsars having periods about few hundred milliseconds. To date, approximately 2000 pulsars are known which are distributed throughout the Milky Way (see, e.g., Lyne \& Graham-Smith 2012 for a review).

The magnetic field of the neutron star can be assumed to be a relic of the field of the progenitor star. Conservation of magnetic flux demands very high field strengths nearby the star, with values in the range $B \approx 10^{6-10} \mathrm{~T}$. The field geometry is bipolar (at least within the light cylinder, i.e., the regime of co-rotation speeds below the speed of light). The combination of 
strong magnetic field plus fast rotation leads to the creation of a strong electric field at the stellar surface, with field strengths up to $E \approx 10^{12} \mathrm{Vm}^{-1}$. The electric field extracts charged particle (electrons, ion) at and around the magnetic poles. The charges propagate along the magnetic field lines at highly relativistic (Lorentz factors $\gamma \approx 10^{7}$ ) energies. Those primary electric charges, plus secondary charges with $\gamma \approx 1000$ originating from electron-positron pair creation, produce synchrotron and (mostly) curvature radiation directed along the magnetic field lines. The emission geometry provides the "lighthouse effect" necessary for the observational pulsar phenomenology. The emitted radiation is partially coherent; the highest flux densities are usually observed at low - few $\mathrm{GHz}$ - radio frequencies (Michel 1991; Beskin et al. 1993).

By geometry, the radiation from pulsars can roughly be approximated as synchrotron radiation from collimated beams of electrons (Section 3.4). Accordingly, one observes (e.g., Rankin 1983) both linear and circular polarization approximately following the pattern outlined in Fig. 3, with details depending on the actual viewing geometry. The angle of linear polarization "swings" through a range of values during a pulse because of the rotation of the star (Michel 1991). Historically, polarimetric observations of the Crab nebula, the supernova remnant surrounding the Crab pulsar, provided the first evidence ever for synchrotron radiation from astronomical objects (Oort \& Walraven 1956).

\subsection{Active Galactic Nuclei}

With luminosities up to approximately $10^{15} L_{\odot}$, active galactic nuclei (AGN; see, e.g., Beckmann \& Shrader 2012 for a recent review) are the most luminous persistent objects in the universe. Their source of energy is the accretion of interstellar matter onto supermassive - meaning $M_{\bullet} \approx 10^{6-10} M_{\odot}-$ black holes which are located in the centers of most, if not all, galaxies. The energy gained from accretion is (largely) radiated away in the form of broad-band continuum emission that is observed from low-frequency radio to high-energy $\gamma$ energies. AGN emission shows strong variability and characteristic statistical properties (e.g., Park \& Trippe 2012; Kim \& Trippe 2013). The radiation from AGN crudely falls into two physical regimes. At low energies ranging roughly from radio to ultraviolet frequencies, the emission is dominated by synchrotron radiation. At higher energies, the radiation is probably produced by inverse Compton scattering of low-energy synchrotron photons.

As AGN are synchrotron sources, their emission is linearly polarized; see also the example provided by Fig. 4. Accordingly, AGN polarization has been studied extensively for several decades and has been used to address the geometries of magnetic fields and the matter distributions (notably particle densities via Faraday rotation) in and around active nuclei (see, e.g., Saikia \& Salter 1988 for an overview). Degrees of linear po- larization are $m_{L} \lesssim 20 \%$, with typical values around $m_{L} \approx 5 \%$ (e.g., Trippe et al. 2010, 2012a). Circular polarization has been observed in a handful of sources on levels $m_{C} \lesssim 1 \%$ (e.g., Agudo et al. 2010).

The outflows of matter from AGN, especially the formation of collimated jets which extend over several megaparsecs in extreme cases, are intimately linked to the immediate (tens of Schwarzschild radii) environment of the central black hole and the geometry of the magnetic fields located there (e.g., Narayan \& Quataert 2005). AGN jets are - largely - optically thin emitters of synchrotron radiation best observable at radio frequencies. Accordingly, linear polarization is used to trace the orientation and strength of magnetic fields along the jets." Observations at multiple wavelengths permit the use of Faraday rotation and Faraday depolarization as a probe of magnetic fields and matter distributions (e.g., Macquart et al. 2006; Taylor et al. 2006; Trippe et al. 2012b). A somewhat unexpected property of AGN jets was the discovery of inverse depolarization - higher degrees of linear polarization at longer wavelengths - in some sources which has been interpreted as a "conspiracy" of spatial small-scale structure and Faraday rotation (e.g., Homan 2012). Occasional observations of circular polarization in AGN jets, most notably in 3C 84 with polarization levels up to $m_{C} \approx 3 \%$, have been attributed to polarization conversion (Homan \& Wardle 2004).

Historically, polarimetric observations of the active Seyfert galaxy NGC 1068 helped to establish the nowadays standard - viewing angle unification scheme of AGN. Spectropolarimetry at optical wavelengths shows that the total flux received from the galaxy is actually composed of two components: one - unpolarized - from directly observable gas with narrow emission lines, one - linearly polarized - from gas with much broader emission lines located within a dust torus and visible only indirectly via Thomson scattering toward the observer (cf., e.g., Baek et al. 2007; Lee 2011). This observation eventually removed the distinction between narrow and broad emission line galaxies which were found to be different realizations of AGN (Miller \& Antonucci 1983; Miller, Goodrich, \& Mathews 1991).

\subsection{Galactic Magnetic Fields}

Disk galaxies and clusters of galaxies are permeated by large-scale (many kpc) magnetic fields with field strengths $B$ on the order of $\mu$ Gauss. In case of disk galaxies, these fields are aligned with the galactic plane and follow closely the galactic structure, especially spiral arms (see, e.g., Fletcher et al. 2011 for an impressive example). The fields are supposed to be generated

\footnotetext{
$\|$ At this point it is important to note that the observed polarization of extended sources is a function of angular resolution: if several individual emitters of polarized radiation fall within the same resolution element (the point spread function or beam of the instrument), the polarization signal can be averaged out partially - a phenomenon known as beam depolarization.
} 
via amplification of primordial cosmic magnetic fields - with $B \sim 10^{-20} \mathrm{G}$ - by "galactic dynamos" driven by galactic rotation. The most widely applied model is the $\alpha-\Omega$ disk dynamo which comprises as parameters (i) the angular speed $\Omega$ of galactic rotation and (ii) the quantity $\alpha=-\tau(\mathbf{v} \cdot \nabla \times \mathbf{v}) / 3$, with $\tau$ being the decorrelation time of plasma turbulences and $\mathbf{v}$ being the plasma velocity (the expression in brackets is also referred to as "kinematic helicity"). In case of galaxy clusters, the fields supposedly originate from extended AGN jets (Section 5.7) which carry strong magnetic fields into the intragalactic medium and where these are dissolved over time (Wielebinski \& Krause 1993; Kulsrud \& Zweibel 2008).

The analysis of large-scale magnetic fields is based on signatures of their interaction with the interstellar or intergalactic medium, specifically:

(i) Faraday rotation (Section 3.8.3) of radiation from pulsars or extragalactic background sources (cf., Clarke 2004; Kronberg 2004; Kronberg \& NewtonMcGee 2011).

(ii) Weak Zeeman effect line splitting, especially the $V(\nu)$ profiles in $\mathrm{H}$ I emission and absorption lines (Section 3.5; cf., Heiles \& Robishaw 2009).

(iii) Polarized synchrotron radiation (Section 3.4; cf., Heald 2009).

(iv) Polarization arising from scattering at magnetically aligned dust grains (Section 3.1.2; cf., Pavel 2011). Notably, this method provided detailed insight into the magnetic field geometry within the center of the Milky Way (Nishiyama et al. 2010) and in parts of the Large Magellanic Cloud (J. Kim et al. 2011).

(v) The Chandrasekhar-Fermi effect (Section 3.8.6; Chandrasekhar \& Fermi 1953)

As should be clear from the discussion provided in Sections 3 and 3.8, (i) and (ii) provide information on magnetic field components along the line of sight, whereas (iii), (iv), and (v) provide information on field components perpendicular to the line of sight.

\subsection{Gamma Ray Bursts}

Gamma-ray bursts (GRB; e.g., Piran 2005; Gehrels et al. 2009) are short, intense pulses of soft (hundreds of $\mathrm{keV}) \gamma$ rays of cosmological origin occurring a few times per day. GRBs last from fractions of a second to hundreds of seconds; with luminosities up to about $10^{46} \mathrm{~W}$ they are among the most luminous (transient) sources of radiation in the universe. According to their duration, GRBs fall into either of two groups:

Long GRBs typically last tens of seconds and are associated with type $\mathrm{Ib} / \mathrm{c}$ supernovae. They are assumed to originate from collapsars, massive evolved stars (probably Wolf-Rayet stars) whose cores collapse into black holes.
Short GRBs usually last less than one second. They are assumed to be caused by mergers of compact objects in binary systems, like two neutron stars or one neutron star and one stellar black hole.

In either case, the outflowing plasma is collimated into relativistic jets with opening angles of a few degrees; this explains the very large apparent isotropic luminosities of GRBs. The GRB emission results from synchrotron and inverse Compton radiation from relativistic electrons. The combination of synchrotron radiation (Section 3.4) and non-isotropic geometry should lead to substantial linear polarization, and, indeed, degrees of polarization up to $30 \%$ have been reported (Götz et al. 2013; Mundell et al. 2013). Sufficient measurement accuracies provided, the polarization can be used to probe the plasma-physical conditions and magnetic fields in GRBs similar to the procedures for AGN (Section 5.7).

As noted by, e.g., Toma et al. (2012), polarized highenergy emission from cosmological sources like GRBs can be used to probe a vacuum birefringence arising from a violation of the Lorentz invariance of Einstein's theory of relativity.

\subsection{Cosmic Background Radiation}

The cosmic microwave background (CMB) is supposed to originate from the hot plasma filling the universe approximately 400000 years after the big bang. To first order, the CMB corresponds to thermal emission from a black body with a temperature of $\approx 2.7 \mathrm{~K}$. Plasma density fluctuations imprint characteristic fluctuations with amplitudes on scales of $\mu \mathrm{K}$ into the angular distribution of the CMB. In addition to fluctuations in the total intensity, one may expect localized linear scattering polarization if the radiation propagating through the plasma shows quadrupole anisotropies - differences in intensities at angles of $90^{\circ}$ in the sky plane. Depending on the underlying geometry, two signatures or modes of polarization have to be distinguished (Zaldarriaga \& Seljak 1997; Kamionkowski, Kosowsky, \& Stebbins 1997).

E mode polarization. A polarization geometry where the orientations of polarization are perpendicular to the gradient of a local perturbation of the CMB is referred to as electric-field like (hence $\mathrm{E}$ ) or gradient mode $(\mathrm{G})$ polarization. By construction, such a polarization pattern does not show a handedness. E mode polarization can be attributed to local energy density fluctuations, also known as scalar perturbations.

B mode polarization. A local curl pattern of polarization with distinct handedness is referred to as magnetic-field like (hence B) or curl mode (C) polarization. The amplitudes of those patterns are supposed to be roughly one order of magnitude weaker than those of $\mathrm{E}$ mode signatures. By geometry, B mode polarization requires tensor perturbations of the CMB. Those perturbations occur due to the propagation of gravitational waves through the CMB plasma; accordingly, 
measurements of B mode polarization are a key experiment for probing primordial gravitational waves and cosmic inflation theories.

In the past decade, E mode polarization has been observed by a variety of ground based CMB telescopes in the approximate frequency range $30-150 \mathrm{GHz}$ (e.g., Leitch et al. 2002; Kovac et al. 2002; Park \& Park 2002; Readhead et al. 2004; Takahashi et al. 2010). A typical CMB telescope is designed as an interferometer with multiple receivers located on a common carrier platform spanning a few meters in diameter. By design (using Rayleigh's criterion for angular resolution) CMB telescopes are sensitive to structures on angular scales of about $1-2^{\circ}$, i.e., the characteristic size scale of $\mathrm{E}$ mode polarization patterns. More recently, the PLANCK satellite has begun a polarization monitoring program aimed at both E and B modes (see, e.g., Lamarre et al. 2003 for technical details), and an observation of B mode polarization by a ground-based CMB telescope has been reported (Hanson et al. 2013).

\section{CONCLUSIONS}

Polarization of light and polarimetry play fundamental roles in astrophysics. Polarization is fundamentally linked to the internal geometry of sources of radiation: the strengths and orientations of magnetic fields, the distribution and orientation of scattering particles like dust grains, the microscopic structure of reflecting surfaces, or intrinsic anisotropies of the primordial plasma filling the early universe. Accordingly, polarimetry has found application in a vast variety of astrophysical fields of study ranging all the way from solar physics to cosmology, comprising even a "personal touch": Understanding the interplay between circularly polarized starlight and the interstellar medium might help to understand the formation of life on Earth.

Reviewing its applications, it is evident that polarimetry is a powerful tool for astrophysics; it provides rich information on the physics of targets that cannot be obtained in any other way. Consequently, a large number of dedicated observational instruments has been constructed and progress is fast. One important current trend is the development of instruments dedicated to polarimetry at the high-energy end of the electromagnetic spectrum, at $\mathrm{X}$ and $\gamma$ ray wavelengths (Section 4.7); another one could be investigations of optical polarimetric interferometry (Elias 2001). Each new technical development eventually opens new windows for observational astronomy. Likewise, more traditional polarimetric techniques profit from the general progress in instrumentation technologies; a key aspect is the improvement of instrumental sensitivities - which have always been harmed by polarimetry measuring relatively small differential fluxes by definition. This being said, we may conclude that polarimetry has the potential for new and exciting astrophysical discoveries in the future.

\section{ACKNOWLEDGMENTS}

I am grateful to Myungshin Im, Masateru Ishiguro, Junghwan OH, TAeseok Lee, Jong-Ho Park, and JaeYoung KIm (all at SNU) for valuable discussion. I acknowledge financial support from the Korean National Research Foundation (NRF) via Basic Research Grant 2012-R1A1A2041387.

\section{REFERENCES}

Agudo, I., Thum, C., Wiesemeyer, H., \& Krichbaum, T. P. 2010, A $3.5 \mathrm{~mm}$ Polarimetric Survey of Radio-Loud Active Galactic Nuclei, ApJSS, 189, 1

Angel, J. R. P. 1974, in Planets, Stars, and Nebulae Studied with Photopolarimetry, Mechanisms that Produce Linear and Circular Polarization, ed. Gehrels, T. (Tucson: Univ. of Arizona Press), 54

Arzoumanian, D., et al. 2011, The Contribution of StarSpots to Coronal Structure, MNRAS, 410, 2472

Bachor, H.-A., \& Ralph, T. C. 2004, A Guide to Experiments in Quantum Optics, 2nd edn. (Weinheim; Wiley$\mathrm{VCH}$ )

Baek, K. M., et al. 2007, Polarization of Thomson Scattered Line Radiation from Broad Absorption Line Outflows in Quasars, JKAS, 40, 1

Beckmann, V., \& Shrader, C. 2012, Active Galactic Nuclei (Weinheim: Wiley VCH)

Bellazzini, R., \& Spandre, G. 2010, in X-Ray Polarimetry: A New Window To Astrophysics, Photoelectric polarimeters, eds. Bellazzini, R., et al. (Cambridge: Cambridge Univ. Press) 19

Berdyugina, S. V., \& Fluri, D. M. 2004, Evidence for the Hanle Effect in Molecular Lines, A\&A, 417, 775

Berdyugina, S. V. 2009, in Cosmic Magnetic Fields: From Planets, To Stars and Galaxies, Proc. IAU Symp. 259, Stellar Magnetic Fields across the H-R Diagram: Observational Evidence, eds. Strassmeier, K. G., et al. (Cambridge: Cambridge Univ. Press), 323

Beskin, V. S., Gurevich, A. V., \& Istomin, Y. N. 1993, Physics of the Pulsar Magnetosphere (Cambridge: Cambridge Univ. Press)

Bloser, P. F., et al. 2010, in X-Ray Polarimetry: A New Window To Astrophysics, The Gamma-Ray Polarimeter Experiment (GRAPE) Balloon Payload, eds. Bellazzini, R., et al. (Cambridge: Cambridge Univ. Press), 314

Born, M., \& Wolf, E. 1999, Principles of Optics, 7th edn. (Cambridge: Cambridge Univ. Press)

Bowell, E., \& Zellner, B. 1974, in Planets, Stars, and Nebulae Studied with Photopolarimetry, Polarizations of Asteroids and Satellites, ed. Gehrels, T. (Tucson: Univ. of Arizona Press), 381

Bradt, H. 2008, Astrophysics Processes (Cambridge: Cambridge Univ. Press)

Brosseau, C. 1998, Fundamentals of Polarized Light: A Statistical Optics Approach (New York: John Wiley \& Sons)

Buenzli, E., \& Schmid, H. M. 2009, A Grid of Polarization Models for Rayleigh Scattering Planetary Atmospheres, A\&A, 504, 259

Burn, B. J. 1966, On the Depolarization of Discrete Radio Sources by Faraday Dispersion, MNRAS, 133, 67 
Campbell, B. A. 2002, Radar Remote Sensing of Planetary Surfaces (Cambridge: Cambridge Univ. Press)

Cawthorne, T. V., \& Wardle, J. F. C. 1988, Kinematic Constraints on Models of the BL Lacertae Object OJ 287 from VLBI Polarization Observations, ApJ, 332, 696

Cellino, A., et al. 2005, A Polarimetric Study of Asteroid 25143 Itokawa, Icarus, 179, 297

Cenacchi, E., Kraus, A., Orfei, A., \& Mack, K.-H. 2009, Full Stokes Polarimetric Observations wth a Single-Dish Radio Telescope, A\&A, 498, 591

Chandrasekhar, S., \& Fermi, E. 1953, Magnetic Fields in Spiral Arms, ApJ, 118, 113

Cho, J., \& Lazarian, A. 2007, Polarization of FIR Emission from T Tauri Stars, JKAS, 40, 113

Clarke, T. E. 2004, Faraday Rotation Observations of Magnetic Fields in Galaxy Clusters, JKAS, 37, 337

Clarke, D. 2010, Stellar Polarimetry (Weinheim: Wiley$\mathrm{VCH})$

Das, H. K., Voshchinnikov, N. V., \& Il'in, V. B. 2010, Interstellar Extinction and Polarization - A Spheroidal Dust Grain Perspective, MNRAS, 404, 265

De Marcellus, P., et al. 2011, Non-Racemic Amino Acid Production by Ultraviolet Irradiation of Achiral Interstellar Ice Analogs with Circularly Polarized Light, ApJL, 727, L27

Dinh-V-Trung. 2009, On the Theory of Astronomical Masers - II. Polarization of Maser Radiation, MNRAS, 399, 1495

Dirac, P. A. M. 1958, The Principles of Quantum Mechanics, 4th edn. (Oxford: Oxford Univ. Press)

Donati, J.-F., \& Landstreet, J. D. 2009, Magnetic Fields of Nondegenerate Stars, ARA\&A, 47, 333

Draine, B. T. 2003, Interstellar Dust Grains, ARA\&A, 41, 241

Dyson, J. E., \& Williams, D. A. 1997, The Physics of the Interstellar Medium, 2nd edn. (New York: Taylor \& Francis)

Edlund, E. 1860, Über die Polarisation des Lichtes der Corona bei totalen Sonnenfinsternissen, AN, 52, 305

Einstein, A. 1905, Über einen die Erzeugung und Verwandlung des Lichtes betreffenden heuristischen Gesichtspunkt, Ann. Phys. (Leipzig), 322, 132

Elias, N. M. II. 2001, Optical Interferometric Polarimetry. I. Foundation, ApJ, 549, 647

Elitzur, M. 1982, Physical Characteristics of Astronomical Masers, Rev. Mod. Phys., 54, 1225

Elitzur, M. 1991, Polarization of Astronomical Maser Radiation, ApJ, 370, 407

Elitzur, M. 2000, in Astrophysical Spectropolarimetry, Astronomical Masers and Their Polarization, eds. TrujilloBueno, J., et al. (Cambridge: Cambridge Univ. Press), 225

Fletcher, A., et al. 2011, Magnetic Fields and Spiral Arms in the Galaxy M51, MNRAS, 412, 2396

Fowles, G. R. 1975, Introduction to Modern Optics, 2nd edn. (New York: Dover Publ.)

Ginzburg, V. L., \& Syrovatskii, S. I. 1965, Cosmic Magnetobremsstrahlung (synchrotron radiation), ARA\&A, 3, 297

Gehrels, N., Ramirez-Ruiz, E., \& Fox, D. B. 2009, GammaRay Bursts in the Swift Era, ARA\&A, 47, 567
Götz, D., et al. 2013, The Polarized Gamma-Ray Burst GRB 061122, MNRAS, 431, 3550

Goldreich, P., \& Kylafis, N. D. 1981, On Mapping the Magnetic Field Direction in Molecular Clouds by Polarization Measurements, ApJ, 243, L75

Goldreich, P., \& Kylafis, N. D. 1982, Linear Polarization of Radio Frequency Lines in Molecular Clouds and Circumstellar Envelopes, ApJ, 253, 606

Goldstein, D. 2003, Polarized Light, 2nd edn. (New York: Marcel Dekker Inc.)

Goodrich, R. W., Cohen, M. H., \& Putney, A. 1995, Spectropolarimetry. II. Circular Polarization Optics and Techniques, PASP, 107, 179

Haken, H., \& Wolf, H. C. 1990, Atom- und Quantenphysik, 4th edn. (Berlin: Springer)

Hamaker, J. P., Bregman, J. D., \& Sault, R. J. 1996, Understanding Radio Polarimetry. I. Mathematical Foundations, A\&ASS, 117, 137

Hamaker, J. P., \& Bregman, J. D. 1996, Understanding Radio Polarimetry. III. Interpreting the IAU/IEEE Definition of the Stokes Parameters, A\&ASS, 117, 161

Hamaker, J. P. 2000, Understanding Radio Polarimetry. IV. The Full-Coherency Analogue of Scalar Self-Calibration: Self-Alignment, Dynamic Range and Polarimetric Fidelity, A\&ASS, 143, 515

Hamaker, J. P. 2006, Understanding Radio Polarimetry. V. Making Matrix Self-Calibration Work: Processing of a Simulated Observation, A\&A, 456, 395

Hanle, W. 1924, Über magnetische Beinflussung der Polarisation der Resonanzfluoreszenz, Z. Phys., 30, 93

Hanson, D., et al. 2013, Detection of B-Mode Polarization in the Cosmic Microwave Background with Data from the South Pole Telescope, Phys. Rev. Lett., 111, 141301

Heald, G. 2009, in Cosmic Magnetic Fields: From Planets, To Stars and Galaxies, Proc. IAU Symp. 259, The Faraday Rotation Measure Synthesis Technique (Cambridge: Cambridge Univ. Press), 591

Heiles, C., Goodman, A. A., McKee, C. K., \& Zweibel, E. 1993, in Protostars and planets III, Magnetic Fields in Star Forming Regions: Observations, eds. Levy, E. H., \& Lunine, J. I. (Tucson: Univ. of Arizona Press), 279

Heiles, C. 2002, in Single-Dish Radio Astronomy: Techniques and Applications, ASP Conf. Series, 278, A Heuristic Introduction to Radio Astronomical Polarization, eds. Stanimirovic̀, S., et al., 131

Heiles, C., \& Robishaw, T. 2009, in Cosmic Magnetic Fields: From Planets, To Stars and Galaxies, Proc. IAU Symp. 259, Zeeman Splitting in the Diffuse Interstellar Medium - The Milky Way and Beyond (Cambridge: Cambridge Univ. Press), 579

Homan, D. C., \& Wardle, J. F. C. 2004, High Levels of Circularly Polarized Emission from the Radio Jet in NGC 1275 (3C 84), ApJL, 602, L13

Homan, D. C. 2012, Inverse Depolarization: A Potential Probe of Internal Faraday Rotation and Helical Magnetic Fields in Extragalactic Radio Jets, ApJL, 747, L24

Huard, S. 1997, Polarization of Light (Paris: Masson)

Hughes, P. A., Aller, H. D., \& Aller, M. F. 1985, Polarized Radio Outbursts in BL Lacertae. II. The Flux and Polarization of a Piston-Driven Shock, ApJ, 298, 301

Ishiguro, M., et al. 1997, Maximum Visible Polarization of 4179 Toutatis in the Apparition of 1996, PASJ, 49, L31 
Jackson, J. D. 1999, Classical Electrodynamics, 3rd edn. (New York: Wiley \& Sons)

Jones, R. C. 1941, A New Calculus for the Treatment of Optical Systems. I. Description and Discussion of the Calculus, J. Opt. Soc. Am., 31, 488

Jordan, S. 2009, in Cosmic Magnetic Fields: From Planets, To Stars and Galaxies, Proc. IAU Symp. 259, Magnetic Fields in White Dwarfs and Their Direct Progenitors, eds. Strassmeier, K. G., et al. (Cambridge: Cambridge Univ. Press), 369

Kamionkowski, M., Kosowsky, A., \& Stebbins, A. 1997, Statistics of Cosmic Microwave Background Polarization, Phys. Rev. D, 55, 7368

Kim, J., et al. 2011, Near-IR Polarimetry around 30 Doradus: I. Separation of the Galactic Sources, JKAS, 44, 135

Kim, K.-T., et al. 2011, 100-GHz Band Test Observations of the KVN 21-m Radio Telescopes, JKAS, 44, 81

Kim, J.-Y., \& Trippe, S. 2013, How to Monitor AGN IntraDay Variability at $230 \mathrm{GHz}, \mathrm{JKAS}, 46,65$

Kitchin, C. R. 2009, Astrophysical Techniques, 5th edn. (Boca Raton: CRC Press)

Kovac, J. M., et al. 2002, Detection of Polarization in the Cosmic Microwave Background Using DASI, Nature, 420, 772

Kronberg, P. P. 2004, New Probes of Intergalactic Magnetic Fields by Radiometry and Faraday Rotation, JKAS, 37, 343

Kronberg, P. P., \& Newton-McGee, K.J. 2011, Remarkable Symmetries in the Milky Way Disc's Magnetic Field, PASP, 28, 171

Kulsrud, R. M., \& Zweibel, E.G. 2008, On the Origin of Cosmic Magnetic Fields, Rep. Prog. Phys., 71, 046901

Kwok, S. 2007, Physics and Chemistry of the Interstellar Medium (Sausalito: University Science Books)

Kwon, Y.-J., \& Suh, K.-W. 2012, Properties of OH, SiO, and $\mathrm{H}_{2} \mathrm{O}$ Masers in O-Rich AGN Stars, JKAS, 45, 139

Kwon, J., et al. 2013, Near-Infrared Circular Polarization Images of NGC 6334-V, ApJL, 765, L6

Lamarre, J. M., et al. 2003, The Planck High Frequency Instrument, a Third Generation CMB Experiment, and a Full Sky Submillimeter Survey, New Astron. Rev., 47, 1017

Landau, L. D., \& Lifschitz, E. M. 1997, Klassische Feldtheorie, 12th edn. (Thun: Harri Deutsch)

Lang, K. R. 2006, Astrophysical Formulae. Vol. I. Radiation, Gas Processes, and High Energy Astrophysics, 3rd edn. (Berlin: Springer)

Lee, H.-W. 2011, Linear Polarization of a Double Peaked Broad Emission Line in Active Galactic Nuclei, JKAS, 44,59

Lee, S.-S., et al. 2011, Single-Dish Performance of KVN 21m Radio Telescopes: Simultaneous Observations at 22 and $43 \mathrm{GHz}$, PASP, 123, 1398

Leitch, E. M., et al. 2002, Measurement of Polarization with the Degree Angular Scale Interferometer, Nature, 420, 763

Lyne, A., \& Graham-Smith, F. 2012, Pulsar Astronomy, 4th edn. (Cambridge: Cambridge Univ. Press)

Macquart, J.-P., et al. 2006, The Rotation Measure and 3.5-millimeter Polarization of Sagittarius A*, ApJ, 646, L111
Mandel, L., \& Wolf, E. 1995, Optical Coherence and Quantum Optics (Cambridge: Cambridge Univ. Press)

Matsumura, M., et al. 2011, Correlation between Interstellar Polarization and Dust Temperature: Is the Alignment of Grains by Radiative Torques Ubiquitous?, PASJ, 63, L43

McConnell, M. L. 2010, in X-Ray Polarimetry: A New Window To AstrophysicsScattering Polarimetry in HighEnergy Astronomy, eds. Bellazzini, R., et al. (Cambridge: Cambridge Univ. Press), 11

Michel, F. C. 1991, Theory of Neutron Star Magnetospheres (Chicago: Univ. of Chicago Press)

Milić, I., \& Faurobert, M. 2012, Hanle Diagnostics of Weak Solar Magnetic Fields, A\&A, 547, A38

Miller, J. S., \& Antonucci, R. R. J. 1983, Evidence for a Highly Polarized Continuum in the Nucleus of NGC 1068, ApJ, 271, L7

Miller, J. S., Goodrich, R. W., \& Mathews, W. G. 1991, Multidirectional Views of the Active Nucleus of NGC 1068, ApJ, 378, 47

Milli, J., et al. 2013, Prospects of Detecting the Polarimetric Signature of the Earth-Mass Planet $\alpha$ Centauri B b with SPHERE/ZIMPOL, A\&A, 556, A64

Müller, H. 1948, The Foundations of Optics (abstract), J. Opt. Soc. Am., 38, 661

Mundell, C. G., et al. 2013, Highly Polarized Light from Stable Ordered Magnetic Fields in GRB 120308A, Nature, 504, 119

Narayan, R., \& Quataert, E. 2005, Black Hole Accretion, Science, 307, 77

Nishiyama, S., et al. 2010, Magnetic Field Configuration at the Galactic Center Investigated by Wide-Field NearInfrared Polarimetry: Transition from a Toroidal to a Poloidal Magnetic Field, ApJL, 722, L23

Oort, J. H., \& Walraven, T. 1956, Polarization and Composition of the Crab Nebula, Bull. Astron. Inst. Neth., 12,285

Ostro, S. J. 1993, Planetary Radar Astronomy, Rev. Mod. Phys., 65, 1235

Ott, T., Eckart, A., \& Genzel, R. 1999, Variable and Embedded Stars in the Galactic Center, ApJ, 523, 248

Pacholczyk, A. G. 1970, Radio Astrophysics, W. H. Freeman \& Co.

Pacholczyk, A. G., \& Swihart, T. L. 1970, Polarization of Radio Sources. II. Faraday Effect in the Case of QuasiTransverse Propagation, ApJ, 161, 415

Pacholczyk, A. G. 1973, Circular Repolarization in Compact Radio Sources, MNRAS, 163, 29P

Park, C.-G., \& Park, C. 2002, Simulation of Cosmic Microwave Background Polarization Fields for AMiBA Experiment, JKAS, 35, 67

Park, J.-H., \& Trippe, S. 2012, Multiple Emission States in Active Galactic Nuclei, JKAS, 45, 147

Pavel, M. D. 2011, Constraining Galactic Magnetic Field Models with Starlight Polarimetry, ApJ, 740, 21

Piran, T. 2005, The Physics of Gamma-Ray Bursts, Rev. Mod. Phys., 76, 1143

Poincaré, H. 1892, Théorie mathématique de la lumière, vol. II (Paris: Georges Carré)

Rankin, J. M. 1983, Toward an Empirical Theory of Pulsar Emission. I. Morphological Taxonomy, ApJ, 274, 333 
Readhead, A. C. S., et al. 2004, Polarization Observations with the Cosmic Background Imager, Science, 306, 836

Reid, M. J., \& Moran, J. M. 1981, Masers, ARA\&A, 19, 231

Rybicki, G. B., \& Lightman, A. P. 1979, Radiative Processes in Astrophysics (New York: Wiley \& Sons)

Saikia, D. J., \& Salter, C. J. 1988, Polarization Properties of Extragalactic Radio Sources, ARA\&A, 26, 93

Saito, H., et al. 2009, Near-Infrared Imaging Polarimetry of S106 Cluster-Forming Region with SIRPOL, AJ, 137, 3149

Sampoorna, M., Nagendra, K. N., \& Stenflo, J. O. 2013, Line-Interlocking Effects on Polarization in Spectral Lines by Rayleigh and Raman Scattering, ApJ, 770, 92

Sault, R. J., Hamaker, J. P., \& Bregman, J. D. 1996, Understanding Radio Polarimetry. II. Instrumental Calibration of an Interferometer Array, A\&ASS, 117, 159

Schrijver, C. J., \& Zwaan, C. 2000, Solar and Stellar Magnetic Activity (Cambridge: Cambridge Univ. Press)

Secchi, P. A. 1860, On the Polarisation of Light Reflected by the Moon, MNRAS, 20, 70

Serkowski, K., Mathewson, D. S., \& Ford, V. L. 1975, Wavelength Dependence of Interstellar Polarization and Ratio of Total to Selective Extinction, ApJ, 196, 261

Silver, E., \& Schnopper, H. 2010, in X-Ray Polarimetry: A New Window To Astrophysics, Bragg Crystal Polarimeters, eds. Bellazzini, R., et al. (Cambridge: Cambridge Univ. Press), 34

Sparks, W., et al. 2012, Remote Sensing of Chiral Signatures on Mars, Planet. Space Sci., 72, 111

Stenflo, J. O. 1982, The Hanle Effect and the Diagnostics of Turbulent Magnetic Fields in the Solar Atmosphere, Solar Phys., 80, 209

Stenflo, J. O. 2013, Horizontal or Vertical Magnetic Fields on the Quiet Sun, A\&A, 555, A132

Stokes, G. G. 1852, Trans. Cambridge Phil. Soc. 9, part III, 399

Sugitani, K., et al. 2010, Near-Infrared Imaging Polarimetry of the Serpens Cloud Core: Magnetic Field Structure, Outflows, and Inflows in a Cluster Forming Group, ApJ, 716,299

Takahashi, Y. D., et al. 2010, Characterization of the BICEP Telescope for High-Precision Cosmic Microwave Background Polarimetry, ApJ, 711, 1141

Taylor, G. B., et al. 2006, Magnetic Fields in the Centre of the Perseus Cluster, MNRAS, 368, 1500

Thompson, A. R., Moran, J. M., \& Swenson, G. W. 2004, Interferometry and Synthesis in Radio Astronomy, 2nd edn. (Weinheim: Wiley-VCH)

Thum, C., Wiesemeyer, H., Paubert, G., et al. 2008, XPOL - the Correlation Polarimeter for the IRAM 30-m Telescope, PASP, 120, 777

Tinbergen, J. 1996, Astronomical Polarimetry (Cambridge: Cambridge Univ. Press)

Toma, K., et al. 2012, Strict Limit on CPT Violation from Polarization of $\gamma$-Ray Bursts, Phys. Rev. Lett, 109, 241104

Townes, C. H. 1997, Astronomical Masers and Lasers, Quantum Electron., 27, 1031

Tribble, P. C. 1991, Depolarization of Extended Radio Sources by a Foreground Faraday Screen, MNRAS, 250, 726
Trippe, S., Paumard, T., Ott, T., et al. 2007, A Polarized Infrared Flare from Sagittarius A* and the Signatures of Orbiting Plasma Hotspots, MNRAS, 375, 764

Trippe, S., Neri, R., Krips, M., et al. 2010, The First IRAM/PdBI Polarimetric Millimeter Survey of Active Galactic Nuclei. I. Global Properties of the Sample, A\&A, 515, A40

Trippe, S., Neri, R., Krips, M., et al. 2012, The First IRAM/PdBI Polarimetric Millimeter Survey of Active Galactic Nuclei. II. Activity and Properties of Individual Sources, A\&A, 540, A74

Trippe, S., Bremer, M., Krichbaum, T. P., et al. 2012, A Search for Linear Polarization in the Active Galactic Nucleus 3C 84 at 239 and $348 \mathrm{GHz}, \mathrm{MNRAS}, 425,1192$

Weisskopf, M. C., et al. 1978, A Precision Measurement of the X-Ray Polarization of the Crab Nebula without Pulsar Contamination, ApJ, 220, L117

Wielebinski, R., \& Krause, F. 1993, Magnetic Fields in Galaxies, A\&AR, 4, 449

Wilson, T. L., Rohlfs, K., \& Hüttemeister, S. 2010, Tools of Radio Astronomy, 5th edn. (Berlin: Springer)

Witzel, G., Eckart, A., Buchholz, R. M., et al. 2011, The Instrumental Polarization of the Nasmyth Focus Polarimetric Differential Imager NAOS/CONICA (NACO) at the VLT, A\&A, 525, A130

Wolstencroft, R. D. 1974, in Planets, Stars, and Nebulae Studied with Photopolarimetry, The Circular Polarization of Light Reflected from Certain Optically Active Surfaces, ed. Gehrels, T. (Tucson: Univ. of Arizona Press), 54

Zaldarriaga, M., \& Seljak, U. 1997, All-Sky Analysis of Polarization in the Microwave Background, Phys. Rev. D, 55,1830

Zeeman, P. 1897, On the Influence of Magnetism on the Nature of the Light Emitted by a Substance, ApJ, 5, 332 